\title{
A mathematical multi-organ model for bidirectional epithelial-mesenchymal transitions in the metastatic spread of cancer
}

\author{
LINNEA C. FRANSSEN* \\ School of Mathematics and Statistics, University of St Andrews, St Andrews, KY16 9SS, UK \\ Current affiliation: Roche, pRED, Translational Modeling \& Simulation, Basel, Switzerland \\ ${ }^{*}$ Corresponding author: 1cf4@st-andrews.ac.uk \\ AND \\ MARK A.J. CHAPLAIN \\ School of Mathematics and Statistics, University of St Andrews, St Andrews, KY16 9SS, UK
}

[Received on 10 May 2020]

\begin{abstract}
Cancer invasion and metastatic spread to secondary sites in the body are facilitated by a complex interplay between cancer cells of different phenotypes and their microenvironment. A trade-off between the cancer cells' ability to invade the tissue and to metastasise, and their ability to proliferate has been observed. This gives rise to the classification of cancer cells into those of mesenchymal and epithelial phenotype, respectively. Additionally, mixed phenotypic states between these two extremes exist. Cancer cells can transit between these states via epithelial-mesenchymal transition (EMT) and the reverse process, mesenchymal-epithelial transition (MET). These processes are crucial both for the local tissue invasion and the metastatic spread of cancer cells. To shed light on the role of these phenotypic states and the transitions between them in the invasive and metastatic process, we extend our recently published multigrid, hybrid, individual-based mathematical metastasis framework (Franssen et al., 2019). In addition to cancer cells of epithelial and of mesenchymal phenotype, we now also include those of an intermediate partial-EMT phenotype. Furthermore, we allow for the switching between these phenotypic states via EMT and MET at the biologically appropriate steps of the invasion-metastasis cascade. We also account for the likelihood of spread of cancer cells to the various secondary sites and differentiate between the tissues of the organs involved in our simulations. Finally, we consider the maladaptation of metastasised cancer cells to the new tumour microenvironment at secondary sites as well as the immune response at these sites by accounting for cancer cell dormancy and death. This way, we create a first mathematical multi-organ model that explicitly accounts for EMT-processes occurring at the level of individual cancer cells in the context of the invasion-metastasis cascade.
\end{abstract}

Keywords: Mathematical oncology; Epithelial-mesenchymal transition; Metastatic spread; Multi-organ model; Tumour microenvironment; Individual-based model.

2000 Math Subject Classification: 34K30, 35K57, 35Q80, 92D25

\section{Introduction}

To elucidate the process by which a subset of cancer cells from a primary tumour invade the local tissue and spread to distant sites in the body, which is also known as the invasion-metastasis cascade, we proposed a first explicitly spatial mathematical modelling framework in Franssen et al. (2019). The framework described the metastatic process by taking into account the spatiotemporal evolution of individual cancer cells. The motivation for developing such a model is the fact that over $90 \%$ of cancer-

(c) The author 2020. Published by Oxford University Press on behalf of the Institute of Mathematics and its Applications. All rights reserved. 
related deaths arise due to metastatic spread rather than as a consequence of tumour growth limited to a primary site. Mathematical models can enhance our understanding of the mechanisms underlying biological phenomena. However, with regards to existing models of the invasion-metastasis cascade, we found the following common short-coming: Metastatic spread is an inherently spatial, cell-based physiolo-gical process. Yet, previous mathematical models did not capture individual cell dynamics during the invasion-metastasis cascade through a spatially explicit approach, e.g. Iwata et al. (2000); Scott et al. (2013); Cisneros \& Newman (2014); Margarit \& Romanelli (2016); Iwata et al. (2000); Benzekry et al. (2016); cf. literature review in Franssen et al. (2019). Biologically accurate computational models of the invasion and the secondary metastatic spread of individual cancer cells could be used in a clinical setting to enhance treatment through patient-specific disease predictions. This is because such models allow to account for e.g. the phenotypic traits of the cells that a tumour consists of, the tumour size and shape, and the tumour microenvironment in a specific patient through the initial conditions of simulations. The in silico simulation results could therefore support clinicians in tailoring treatment to each patient by predicting the individual's disease evolution, and by testing and optimising treatments in an ethical, time-effective approach.

To develop a model that will ultimately be used to inform clinicians with regards to cancer treatment decisions, in the concluding section of Franssen et al. (2019) we proposed several features to enhance the base modelling framework developed therein. Inter alia, we concluded that-since epithelialmesenchymal transition (EMT) and mesenchymal-epithelial transition (MET) are major factors in the metastatic process - a first natural extension to the modelling framework would be their inclusion. This is achieved in this paper by accounting for phenotypic switching to represent permanent and transient transitions between the epithelial and the mesenchymal phenotypic state as well as a mixed epithelial/mesenchymal state, as biologically observed during the invasion-metastasis cascade (Celià-Terrassa et al., 2012). Thus, EMT-related features are introduced to the existing metastasis framework to accurately represent their role in the invasion-metastasis cascade. We also account for differences in the extracellular matrix (ECM) density of the tissue of the primary and secondary tumour growth sites involved in our simulations. This way, we address another enhancement to the model suggested in Franssen et al. (2019) by taking a further step towards developing a biologically accurate multi-organ spatially explicit model. Finally, we include the immune response at secondary sites through the modelling of dormancy and death of metastasised cancer cells.

The remainder of the paper is organised as follows. In Section 2, we introduce the biological background of EMT, MET and of the cancer cell phenotypes involved in the context of the invasionmetastasis cascade. In Section 3, we describe how EMT and MET-which we may for simplicity also jointly refer to as the EMT process throughout this paper-are included in our general mathematical modelling framework of metastatic spread. As part of this, we give an overview of previous models concerned with EMT in cancer invasion at the beginning of this section. In Section 4, we outline how the computational simulations are set up. In Section 5, we present the simulation results. Finally, in Section 6 , we discuss how the results fit in with current biological findings and hypotheses. We also give an overview of future work.

\section{Biological background}

Mutations of key genes in only a few epithelial cells in the body can ultimately lead to the formation of carcinomas, which are the group of solid tumours arising from epithelial tissues in the body. Abnormally rapid proliferation caused by these mutations can result in the formation of an avascular tumour with a diameter of up to approximately $0.1-0.2 \mathrm{~cm}$ (Folkman, 1990). Nutrient and oxygen supply to tumour 


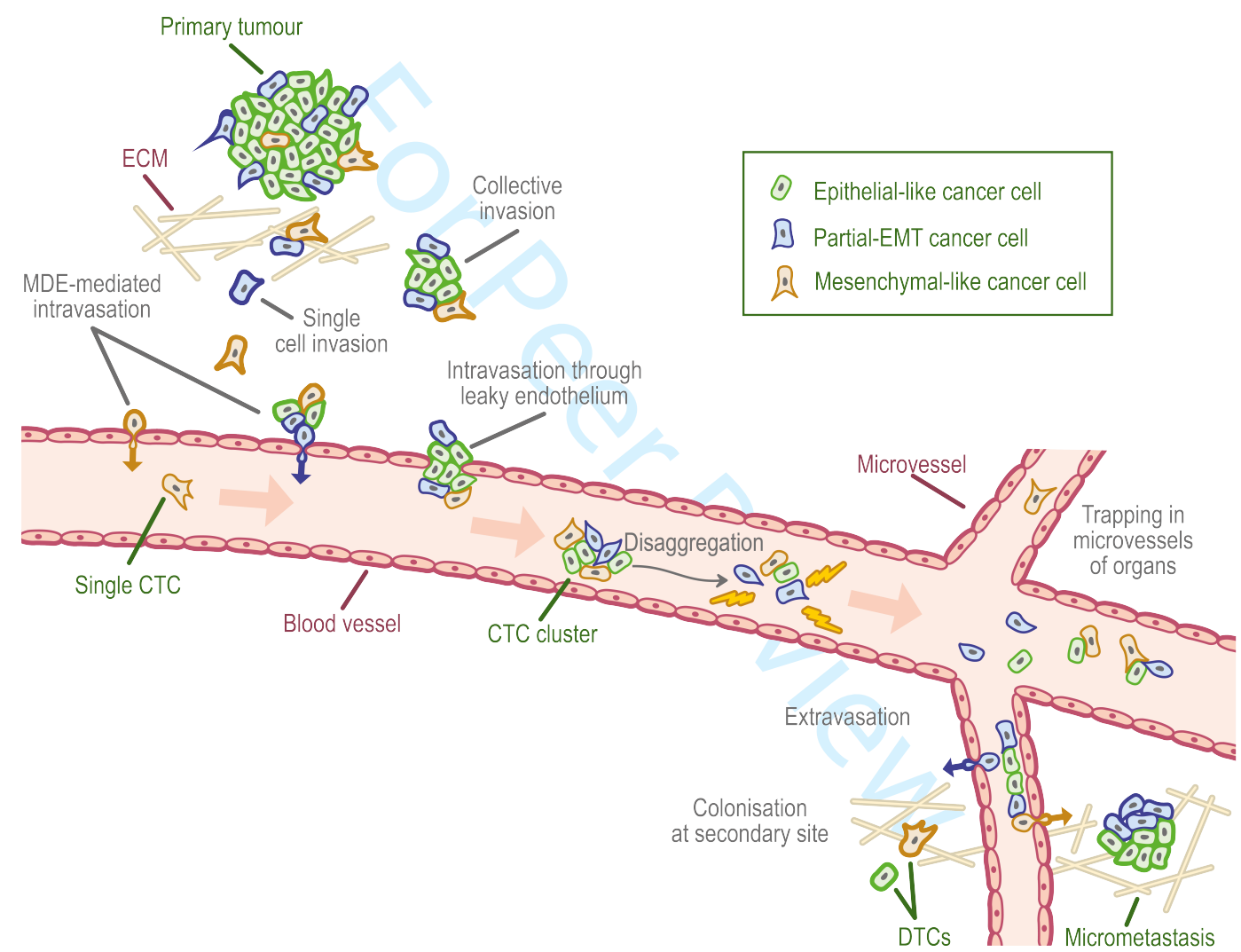

Figure 1. Schematic overview of the invasion-metastasis cascade. Single mesenchymal-like and partial-EMT cancer cells as well as heterogeneous clusters consisting of mesenchymal-like, partial-EMT and epithelial-like cancer cells break free from the primary tumour and invade the surrounding tissue (top left). They can intravasate via active matrix-degrading enzyme (MDE)mediated and passive mechanisms (mid-left, along epithelium of the vessel). Once in the vasculature, circulating tumour cell (CTC) clusters may disaggregate (centre) and CTCs may die. Surviving cells may extravasate through the walls of the microvasculature to various secondary sites in the body (bottom right). Successful colonisation there can result in either disseminated tumour cells (DTCs) or in micrometastases, which have the potential to develop into full-blown metastases. 
cells in this early avascular stage occurs via diffusion from a vessel source only. The diffusion limit of oxygen is $100-200 \mu \mathrm{m}$. Hence, vascular growth is restricted by the metabolic needs of the cells forming the rapidly expanding tumour. It has been observed that, once the tumour growth limit of the avascular phase is reached, a subset of cancer cells starts invading the tissue surrounding the tumour either as individual cancer cells or as cancer cell clusters. These invading cells continue to perform random motion but additionally are driven away from the primary tumour mass by gradients in nutrients, oxygen and in the extracellular matrix (ECM) density. Furthermore, the cancer cells secrete chemicals, collectively known as tumour angiogenic factors (TAFs), which start recruiting new blood vessels (Folkman \& Klagsbrun, 1987) - a process known as (tumour-induced) angiogenesis. The resulting newly established vasculature enables the transport of nutrients and oxygen required for further tumour growth. Also, cancer cells may now intravasate into the newly grown blood vessels, travel through the bloodstream and extravasate at distant sites in the body where space and nutrients are less of a limiting factor to growth. The successful relocation of cancer cells from a primary location to a secondary location in the body via the described sequence of steps of the invasion-metastasis cascade is known as metastatic spread. Successfully extravasated cancer cells occur either as single disseminated tumour cells (DTCs) or as small clusters of cancer cells, called micrometastases. The majority of micrometastases and, even more so, of DTCs die due to maladaptation to the new microenvironment as well as due to the local immune response (Aceto et al., 2014). However, surviving cancer cells may proliferate, which can lead to the formation of secondary tumours, called metastases, at sites in the body away from the primary tumour. Other DTCs and micrometastases may remain dormant at first but have the potential to proliferate into vascularised metastases at the secondary sites at some later point in time. The full process described here is shown schematically in Figure 1. The corresponding biological background is further explained in Franssen et al. (2019) by considering each of the steps in the invasion-metastasis cascade-i.e. cancer cell invasion, intravasation, vascular travel, extravasation and regrowth at new sites in the body-in turn.

Cancer cells adapt to the environmental requirements of the various steps of the invasion-metastasis cascade via changes in phenotype (Jolly et al., 2017a). EMT and MET are a canonical group of —at least transiently-observed phenotypic changes that are assumed to be crucial for metastatic spread (Guo et al., 2012; Ye et al., 2015; Krebs et al., 2017). Various combinations of so-called EMT-inducing transcription factors (EMT-TFs) together with a number of extracellular molecules in the tumour microenvironment and related pathways are thought to trigger EMT (Jie et al., 2017). The cell-cell adhesion between formerly epithelial-like cancer cells is typically reduced upon activation of EMT. At the same time, the cancer cells tend to express more cell-matrix adhesion enhancing molecules like N-cadherin (Micalizzi et al., 2010). As part of this combination of changes, the characteristic polygonal cobblestone-like cell shape of epithelial cells is progressively replaced by a spindle-shaped morphology, as shown on the right of Figure 2. Also, the motility and invasiveness of the cancer cells are enhanced (Jie et al., 2017; Dongre \& Weinberg, 2019). As another result of EMT, the cells become increasingly potent at degrading the underlying basement membranes of organs and vessels as well as the ECM via the expression of metalloproteases (MMPs) (Dongre \& Weinberg, 2019). As a trade-off, they become less proliferative. MET can reverse the phenotypic changes induced by EMT, thus-generally speaking - causing the cells to become less motile and invasive while enhancing their proliferative potential.

Traditionally, the EMT-process has been viewed to result in cells of epithelial and of mesenchymal phenotype in a binary sense (Pastushenko \& Blanpain, 2018; Dongre \& Weinberg, 2019). Yet, more recently, intermediate states - commonly referred to as hybrid, incomplete or partial-EMT states-on the spectrum between the fully epithelial and fully mesenchymal state have been shown to exist in 

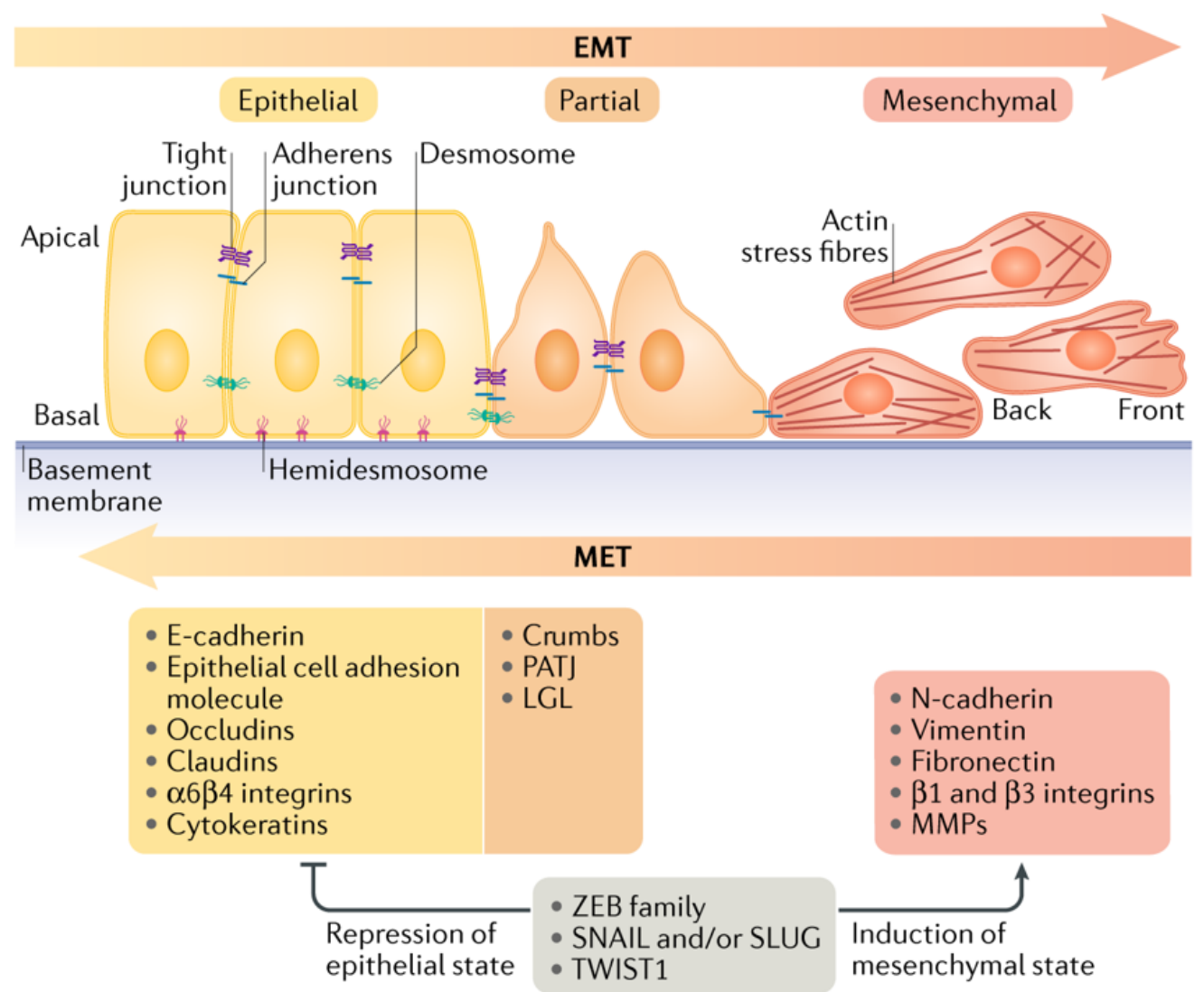

Figure 2. Typical EMT transition states in carcinoma progression and metastatic spread. During carcinoma invasion and metastatic spread, the formerly epithelial phenotype of cancer cells (left) can—via the processes of partial or full EMT—evolve to a partial-EMT (middle) or a mesenchymal (right) phenotype. Generally, the further to the right of the EMT spectrum a cell is located, the less it attaches to other cells. The trade-off for this gain in motility is a decreased proliferative potential. MET is the reverse process. It is indicated by the arrow along the bottom of the figure. Reproduced from Dongre \& Weinberg (2019) with permission from Nature Publishing Group. 
various cell lines of patient xenografts and of human primary cancers, such as breast, head and neck, and pancreatic cancer (Pastushenko \& Blanpain, 2018). Cancer cells in these intermediate phenotypic states are assumed to show a variety of combinations of the above-mentioned phenotypic traits. The full transition from an epithelial to a mesenchymal state, which had formerly been assumed to be the only possible outcome of EMT, has recently been shown to actually be rare during carcinogenesis (Dongre $\&$ Weinberg, 2019). Furthermore, cell cycle arrest may occur in fully mesenchymal cancer cells (Vega et al., 2004; Lovisa et al., 2015), while partial-EMT cancer cells continue to be able to proliferate (Handler et al., 2018).

In what follows, the roles of EMT and MET as well as of epithelial-like, partial-EMT and mesenchymal-like cancer cells during the various steps of the invasion-metastasis cascade are elucidated in more detail. The five steps of the invasion-metastasis cascade are printed in bold for better orientation. A more in-depth description of the EMT-unrelated features of these steps of the invasion-metastasis cascade is provided in Franssen et al. (2019).

Local cancer invasion Carcinomas are tumours that arise from epithelial tissue. However, cancer cells have been found to either invade as single cells of partial-EMT or of mesenchymal phenotype or as clusters, which often consist of cancer cells of heterogeneous phenotypes (Friedl \& Wolf, 2003). Hence, EMT of some degree - at least in a subset of the cancer cells - at the primary site is a prerequisite for this first step of the invasion-metastasis cascade (Francart et al., 2018; Pastushenko \& Blanpain, 2018). Migrating cells usually employ their acquired mesenchymal traits, i.e. the decrease or loss in cell-cell adhesion and increases in cell-ECM adhesion and in matrix-degrading enzyme (MDE)-expression, to invade (Friedl \& Wolf, 2003; Bill \& Christofori, 2015). This hypothesis is, for example, supported by reports suggesting that invading cancer cell clusters contain cells that have undergone partial EMT in vivo (Tsai et al., 2012; Ocaña et al., 2012). Moreover, the occurrence of clusters highlights that partial EMT allows for the cancer cells to maintain at least some aspects of the epithelial cell-cell adhesion (Cheung \& Ewald, 2016). Furthermore, the spatial location of cancer cells of partial-EMT and of epithelial phenotype was investigated by Puram et al. (2017) in situ in oral cavity head and neck squamous cell carcinomas (HNSCCs). Using immunohistochemistry to stain a collection of tumours, they found that, while the core of the tumours contained malignant cells of epithelial phenotype, partial EMT had occurred in the cancer cells at the leading tumour edge in the proximity of cancer-associated fibroblasts (CAFs) in the tumour microenvironment. A corresponding explanation in the form of a diagram and a stained tissue sample is shown in Figure 3.

Intravasation As explained in detail in Franssen et al. (2019), unless vessels are ruptured-for instance due to the tumour microenvironment-modulating effects of EMT-TFs (Jolly et al., 2017b) and subsequently opportunistically entered by cancer cells, MDE-expressing cancer cells are required to allow the intravasation of cancer cells into the vasculature. Therefore, epithelial-like cancer cells cannot gain access to undamaged vessels, while partial-EMT and mesenchymal-like cancer cells can (Jolly et al., 2018). Similarly, cancer cell clusters that consist at least partially of partial-EMT or mesenchymallike cancer cells can enter undamaged vessels using MDEs.

Travel through the vasculature The majority of circulating tumour cells (CTCs) and CTC clusters in the vasculature were found to be of partial-EMT phenotype (Jolly et al., 2018). Moreover, Armstrong et al. (2011) found that in women with metastatic breast cancer and men with castration-resistant prostate cancer more than $75 \%$ and $80 \%$ of CTCs, respectively, co-expressed epithelial and mesenchymal markers. Similarly, studies by Thiery \& Lim (2013) and by Yu et al. (2013) reported that a significant proportion of CTCs was of partial-EMT or mesenchymal-like phenotype in patients with metastatic breast cancer. CTCs of partial-EMT phenotypes have further been observed in the blood of patients with cancer of the liver, prostate and lungs as well as in patients with colorectal, nasopharyngeal and gas- 

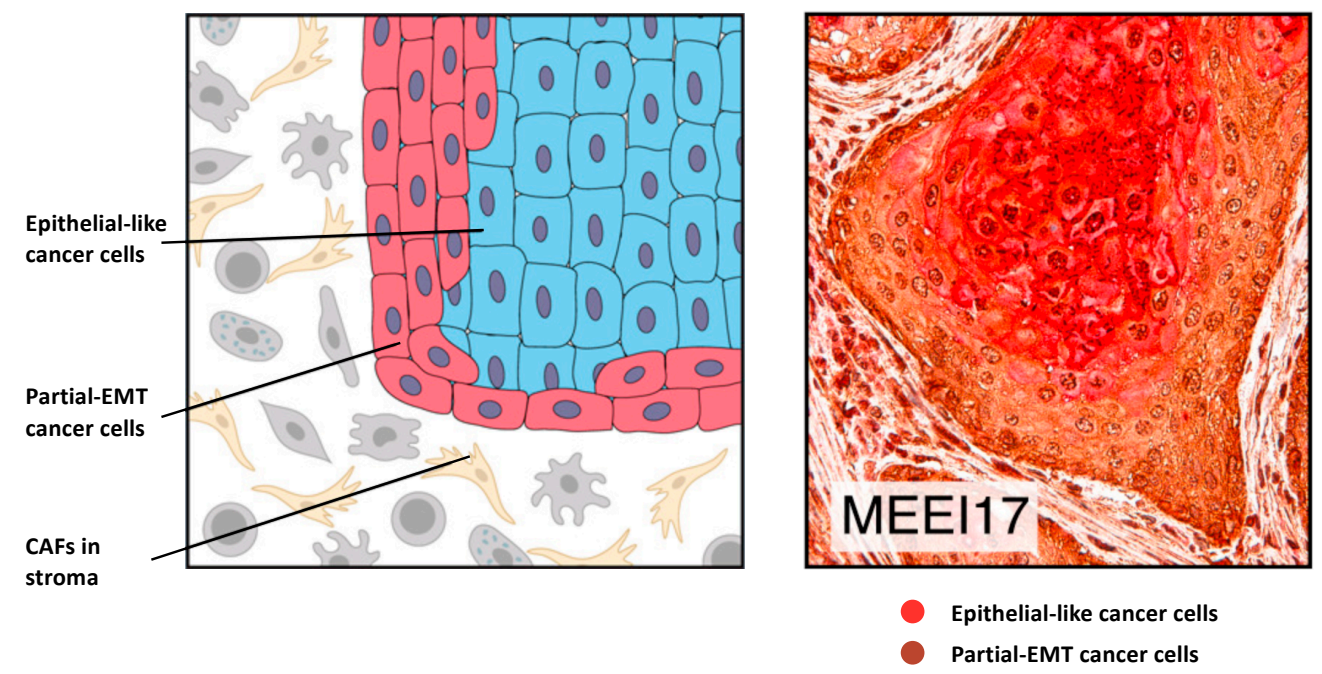

Figure 3. Partial EMT occurs at leading tumour edge in head and neck squamous cell carcinoma (HNSCC). In situ spatial location of cancer cells expressing a partial EMT programme versus those of epithelial phenotype within HNSCC tumours, both schematically (left) and in human tissue (right). On the right, immunohistochemistry was used to stain the tumour for PDPN, one of the top genes in the partial-EMT programme, as well as for SPRR1B, an epithelial differentiation marker. Partial-EMT cancer cells were located at the leading edge of tumours in proximity to cancer-associated fibroblasts (CAFs) in the surrounding stroma, epithelial-like cancer cells at the core of tumours. Reproduced from Puram et al. (2017) with permission from Elsevier.

tric cancer. In these types of cancer, the partial-EMT phenotype correlates with poor clinical prognosis when compared to the occurrence of cancer cells of purely epithelial or purely mesenchymal phenotype (Pastushenko \& Blanpain, 2018). The prominence of partial-EMT cancer cells at the tumour edge as well as their ability to intravasate into the vasculature using MDEs offer potential explanations for these findings. An additional explanation is that at least a subset of partial-EMT CTCs is more resistant to anoikis, i.e. to apoptosis induced by lack of correct cell-ECM attachment (Huang et al., 2013). However, independent of phenotype, both single CTCs and CTC clusters are exposed to physical stresses and to attacks by natural killer cells in the vasculature. One consequence is that only a small number of cancer cells shed from the primary tumour actually reach the microvasculature at metastatic sites. Another effect of this is the (partial) disaggregation of cancer cell clusters, as shown in the centre of Figure 1. This leads to smaller CTC clusters and to an increased number of single CTCs.

Extravasation For the subset of CTCs and CTC clusters that do reach the microvessels at distant organs, extravasation, i.e. the translocation from the vasculature to the tissue at a secondary site, is the next step. During extravasation, the phenotype of the cancer cells is believed to play a tangential role at most - CTCs of all phenotypes appear to be able to extravasate (Banyard \& Bielenberg, 2015) with the aid of mechanisms explained further in Lambert et al. (2017); Franssen et al. (2019).

Colonisation and metastatic growth Cancer cell phenotypes are, once again, of crucial importance when it comes to the colonisation and metastatic growth of cancer cells at the secondary sites. Also, EMT alone fails to explain this last step of the invasion-metastasis cascade, given that macrometastases in humans often present similar histopathological traits to the primary tumours they originate 
from. These traits include a mainly epithelial-like morphology (Pastushenko \& Blanpain, 2018) with a relatively small subset of cancer cells with phenotypes further along the EMT spectrum (Dongre \& Weinberg, 2019) — despite the above-described evidence of the abundance of partial-EMT CTCs in the vasculature. Consequently, this suggests that some degree of MET is needed for macrometastatic growth. A murine prostate cancer model by Ruscetti et al. (2015) delivers insight into this. Cancer cells in macrometastases that had spread to the lungs were found to have mainly epithelial markers and few mesenchymal markers; the inverse constitution was found in dormant micrometastatic lesions. Coherently, in a study by Ocaña et al. (2012), it was proposed that the constant overexpression of the EMT-inducer PRRX1 in human breast tumour cell lines, which were injected intravenously into chick embryos, may lock cancer cells in a mesenchymal-like phenotypic state. This was suggested to inhibit the cells from performing MET, which, in turn, failed to give rise to lung metastases. Similarly, Kröger et al. (2019) concluded from several studies that a stable mesenchymal-like phenotype without any MET potential cannot succeed in metastatic re-seeding.

Finally, as elaborated in Franssen et al. (2019), it is noteworthy that experimental evidence suggests that less than $0.07 \%$ of all initially intravasated single CTCs form micrometastases and less than $0.018 \% \pm 0.017 \%$ form macrometastases 13 days after intravasation (Luzzi et al., 1998; Valastyan \& Weinberg, 2011). Maladaptation to the new tumour microenvironment, with the consequence that only a few cells are able to proliferate, is regarded to be a main contributor to the poor survival at secondary sites (Dongre \& Weinberg, 2019). Compared to single CTCs, CTC clusters were described to have 23 to 50 times the metastatic potential (Aceto et al., 2014). One explaining factor for this is that heterogeneity in the cell phenotype, as often found in such clusters, can be advantageous during colonisation (Jolly et al., 2018).

\section{The EMT/MET multi-organ extension of the metastasis modelling framework}

In this section, the inclusion of EMT-related processes into the recently introduced mathematical framework for the modelling of the metastatic spread of cancer by Franssen et al. (2019) is outlined. Only new EMT-related features will be established here-the reader is referred to Appendix A to consult the existing underlying metastasis modelling framework, onto which we impose the alterations described in this section. Further, we introduce changes to the existing framework that allow us to differentiate between the cell behaviour on the various organs as well as to account for dormancy and death of metastasised cancer cells as a result of the potential immune response at and maladaptation to secondary sites. We begin by giving an overview of existing mathematical models that include, in the wider sense, EMT-related features in the context of spatially explicit cancer invasion. A review of such mathematical models of the EMT-process in the context of metastasis will be omitted as, to our knowledge, this paper is the first metastasis model to include the roles of EMT and MET, and of the corresponding phenotypes of individual cancer cells in a spatially explicit manner.

Andasari et al. (2011) extended and analysed a system of equations initially proposed in Chaplain \& Lolas (2005) to represent the interaction between cancer cells, the MDE urokinase-type plasminogen activator (uPA), uPA inhibitors of type PAI-1, the ECM-cleaving and MMP-activating enzyme plasmin, and the ECM component vitronectin. They allowed for cancer cells to mutate into a phenotype that diffuses, migrates and proliferates more rapidly, which was modelled using a Heaviside function. While the current biological evidence on EMT-related phenotypic changes somewhat contradicts the notion of such a 'go-and-grow' mutation, the proposed model was an important step towards the inclusion of mutations in cell phenotype in spatial cancer invasion models. Gerisch \& Chaplain (2008) modified the local haptotaxis-based partial differential equation (PDE) model proposed in Anderson et al. (2000) to 
include cell proliferation and ECM remodelling as well as cell-matrix and cell-cell adhesion. This was achieved using an integro-differential PDE model, which incorporated cell-cell adhesion using integral terms. Domschke et al. (2014) extended this model further. In particular, they introduced a subpopulation of cancer cells that arose from the initial cell population by mutation, again by using a Heaviside function. The mutation resulted in a decrease in self-adhesion of the cancer cells and an increase in cell-matrix adhesion, which caused the mutated cancer cells to spread more rapidly into the surrounding tissue. This is coherent with the current biological understanding that EMT causes more invasive phenotypes. In order to include physiological mechanisms that lead to EMT, Hellmann et al. (2016) modelled EMT from differentiated cancer cells to cancer stem cells (CSCs), which have biological properties comparable to the epithelial-like and the mesenchymal-like cancer cells in our model, respectively. In this approach, EMT was triggered by epidermal growth factors (EGFs) in the ECM. Subsequently, an advection-reaction-diffusion system of Keller-Segel taxis type was used to study the invasion of both types of cancer cells into the ECM. Numerical simulations were proposed as a proof of concept to show that combining the two systems can account for EMT in a biologically accurate manner. Sfakianakis et al. (2017) developed this model of EGF-driven EMT further. In the corresponding simulations, the detachment of CSCs from the main tumour body of differentiated cancer cells-due to their ability to invade the tissue comparatively more rapidly - was reproduced qualitatively. More recently, Sfakianakis et al. (2018) introduced a coupled two-dimensional hybrid system that governed the spatiotemporal evolution of individual mesenchymal cancer cells by a system of stochastic differential equations (SDEs), while the collectively moving epithelial cancer cells, the ECM and the MMPs evolved according to PDEs. This novel modelling technique considered the effects of EMT and MET on cancer invasion using phase transition operators. As a result, the in silico invasion assays simulated using the Sfakianakis et al. (2018) approach presented 'islands' of invading cancer cells ahead of the expanding initial main cancer cell mass, which had arisen from EMT and subsequent MET. These 'islands' away from the tumour mass are frequently observed in vivo but do not typically present themselves in solely macroscopic or atomistic cancer invasion models.

From the literature review, we draw several conclusions. Firstly, as far as we know, no spatially explicit model that describes the role of EMT and MET in metastatic spread-as opposed to their role in invasion alone-exists. Consequently, none of the existing models capture the site- and locationdependent occurrence of EMT and MET in all of the steps of the invasion-metastasis cascade-i.e. in cancer cell invasion, intravasation, vascular travel, extravasation and during regrowth at new sites in the body - in a spatial manner. Secondly, to our knowledge, the simulations from existing spatiotemporal ECM invasion models that account both for epithelial from mesenchymal cancer cell populations as well as for the transition between the phenotypic states, such as Domschke et al. (2017), lack the inclusion of intermediate partial-EMT phenotypes. Yet, it has recently become evident that cancer cells of partialEMT phenotype are crucial to the EMT process, as explained in Section 2. With the aim of closing the current gap in the literature, we propose an extension to the spatially explicit hybrid modelling framework in Franssen et al. (2019). The resulting model describes the invasive growth dynamics both of the primary tumour by - inter alia — accounting for EMT, as well as growth in the early avascular stages at potential secondary metastatic sites by accounting for MET. Additionally, transport from primary to secondary sites is modelled. In what follows, we introduce the ideas and assumptions that the EMT extension of the metastasis framework builds on.

As Franssen et al. (2019), we use $G+1$ non-overlapping spatial domains-one to represent the primary tumour site, $\Omega_{\mathrm{p}} \subset \mathbb{R}^{2}$, and $G \in \mathbb{N}$ spatial domains representing the sites of potential secondary metastatic spread, $\Omega_{\mathrm{s}}^{a} \subset \mathbb{R}^{2}$, where $a=1,2, \ldots, G$. As previously, we represent the MMP-2 
10 of 40

Local cancer cell invasion

Intravasation

Travel through the vasculature

Extravasation

Metastatic growth
L.C. FRANSSEN AND M.A.J. CHAPLAIN

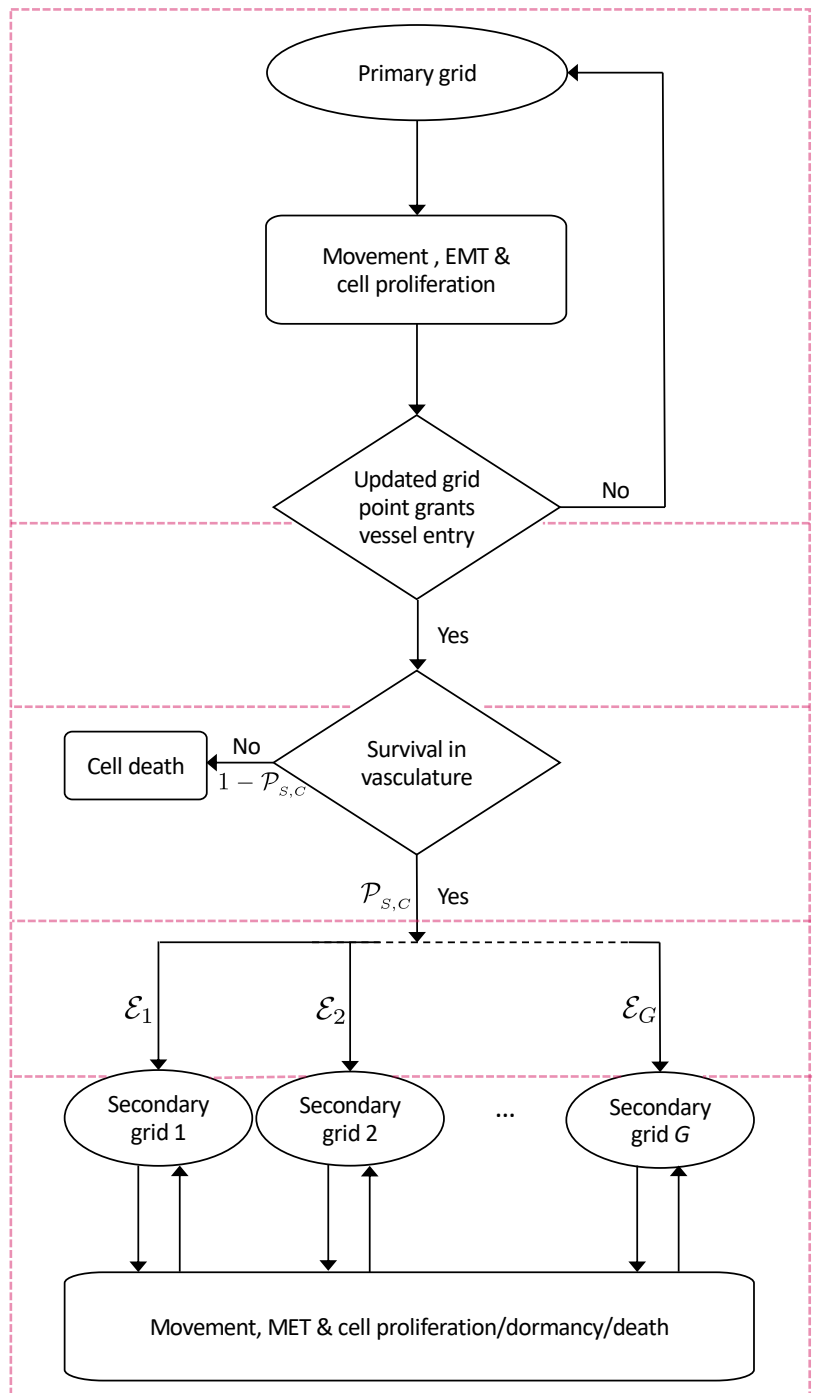

Figure 4. Flowchart of the extended invasion-metastasis hybrid model. At each time step, each cancer cell on the primary grid may move, may perform EMT with some (location-dependent) probability and may proliferate as explained in detail in the text. A cancer cell remains on the primary grid during the respective time step, unless it is placed on a grid point that represents a blood vessel. In the latter case, single CTCs and CTC clusters may enter the vasculature. They spend a number of time steps in the circulation and survive with a probability of $\mathscr{P}_{S}=\mathscr{P}_{\mathrm{E}}, \mathscr{P}_{\mathrm{M}}$ or $\mathscr{P}_{\mathrm{E} / \mathrm{M}}$ in the case of single CTCs of epithelial, mesenchymal and partial-EMT phenotype, respectively, and with a probability of $\mathscr{P}_{C}$ in the case of CTC clusters. Cancer cells that do not survive are removed from the simulation. Surviving CTCs and CTC clusters are placed onto one of $G$ secondary grids with the respective probability $\mathscr{E}_{1}, \mathscr{E}_{2}, \ldots, \mathscr{E}_{G}$. Cancer cells on the secondary grids move and proliferate like cancer cells on the primary grid (potentially with different parameter values to represent organ- and patient-specific differences in the local tumour microenvironment). However, partial-EMT cells may now revert to cells of an epithelial phenotype via MET and there furthermore exists a probability for cell death and dormancy. For better orientation, the red boxes with their labels on the left correspond to the sections indicated in bold in Sections 2 and 3 of the text as well as in Appendix A. 
concentration and the ECM density at position $\vec{x}$ at time $t$ in these spatial domains by the continuous functions $m(t, \vec{x})$ and $w(t, \vec{x})$, respectively, while capturing the spatiotemporal evolution of epithelial, partial-EMT and mesenchymal cancer cells as well as of the membrane-bound MT1-MMP in a discrete approach, $c f$. Anderson \& Chaplain (1998); Anderson et al. (2000); Franssen et al. (2019). Also analogously to Franssen et al. (2019), we allow cancer cells to travel from primary to secondary sites via the vasculature by designating locations in the primary spatial domain to function as entry points into blood vessels and, similarly, we impose a spatial map of exit locations from the vasculature onto the secondary metastatic domains.

The EMT-related features that are novel to the metastatic framework are explained according to which key step of the invasion-metastasis cascade-i.e. cancer cell invasion, intravasation, vascular travel, extravasation and metastatic growth - they belong to. To enhance the clarity of presentation, as Franssen et al. (2019), we begin each paragraph by printing the description of corresponding the step in bold. We also label the respective sections in the flowchart presented in Figure 4, which visually describes the model, accordingly.

Local cancer cell invasion As explained in detail in Franssen et al. (2019), the evolution of the MMP-2 concentration and of the ECM density are modelled in a continuum approach. To account for the inclusion of partial-EMT cancer cells in our model, we extend equations (A.1) and (A.2) from the former model slightly. Accordingly, the spatiotemporal evolution of the MMP-2 concentration $m(t, \vec{x})$ is given by

$$
\frac{\partial m}{\partial t}=\overbrace{D_{m} \nabla^{2} m}^{\text {diffusion }}+\overbrace{\Theta_{\mathrm{M}} c_{\mathrm{M}_{n}}+\Theta_{\mathrm{E} / \mathrm{M}} c_{\mathrm{E} / \mathrm{M}_{q}}}^{\text {expression }}-\overbrace{\Lambda m}^{\text {decay }},
$$

along with zero-flux boundary conditions. Here, $c_{\mathrm{M}_{n}}, n=0,1,2, \ldots, \mathscr{Q}$, and $c_{\mathrm{E} / \mathrm{M}_{q}}, q=0,1,2, \ldots, \mathscr{Q}$, with $n+q \leqslant \mathscr{Q}$, denote the presence of $c_{\mathrm{M}_{n}}$ mesenchymal-like cancer cells and $c_{\mathrm{E} / \mathrm{M}_{q}}$ partial-EMT cancer cells - totalling no more than the preferred carrying capacity $\mathscr{Q}$ - at a given position $\vec{x}$, following the notation by Stéphanou et al. (2006) and McDougall et al. (2012). $D_{m}>0$ is the MMP-2 diffusion coefficient, and $\Theta_{M}>0$ and $\Theta_{E / M}>0$ are the rates of MMP- 2 concentration provided by mesenchymallike cancer cells and the partial-EMT cancer cells, respectively. Consequently, $\Theta_{\mathrm{M}} c_{\mathrm{M}_{n}}$ and $\Theta_{\mathrm{E} / \mathrm{M}} c_{\mathrm{E} / \mathrm{M}_{q}}$ represent the local expression of MMP-2 by the mesenchymal-like and the partial-EMT cancer cells, respectively. Finally, $\Lambda>0$ is the rate at which MMP-2 decays. Note that the mesenchymal-like and partial-EMT cancer cells also express MT1-MMP. However, MT1-MMP acts only locally where it is bound to the cancer cell membrane and its spatiotemporal evolution is hence congruent to that of the mesenchymal-like and of the partial-EMT cancer cells. Therefore, we do not include a separate equation.

Both the MT1-MMP expressed on the membranes of the mesenchymal-like and the partial-EMT cancer cells and the diffusive MMP-2 that these cells secrete degrade the ECM. In the respective equation (3.2), this is expressed through the degradation rates $\Gamma_{\mathrm{M}}>0$ and $\Gamma_{\mathrm{E} / \mathrm{M}}>0$ in the case of the MT1-MMP bound to the membranes of partial-EMT and of mesenchymal-like cancer cells, respectively, and for the diffusive MMP-2 by the degradation rate $\Gamma_{m}>0$. Hence, given that we are disregarding ECM-remodelling for simplicity, the evolution of the ECM density $w(t, \vec{x})$ is governed by the following PDE: 


$$
\frac{\partial w}{\partial t}=-\overbrace{\left(\Gamma_{\mathrm{M}} c_{\mathrm{M}_{n}}+\Gamma_{\mathrm{E} / \mathrm{M}} c_{\mathrm{E} / \mathrm{M} q}+\Gamma_{m} m\right) w}^{\text {degradation }},
$$

along with no-flux boundary conditions.

For the cancer cell migration on the grid we adopt a discrete approach where the movement probabilities of the cancer cells are given as follows:

$$
\begin{aligned}
& \mathscr{P}_{0}: \mathscr{P}_{i-1, j}^{n}:=\frac{\Delta t}{(\Delta x)^{2}}\left[D_{k}-\frac{\Phi_{k}}{4}\left(w_{i+1, j}^{n}-w_{i-1, j}^{n}\right)\right], \\
& \mathscr{P}_{1}: \mathscr{P}_{i+1, j}^{n}:=\frac{\Delta t}{(\Delta x)^{2}}\left[D_{k}+\frac{\Phi_{k}}{4}\left(w_{i+1, j}^{n}-w_{i-1, j}^{n}\right)\right], \\
& \mathscr{P}_{2}: \mathscr{P}_{i, j+1}^{n}:=\frac{\Delta t}{(\Delta x)^{2}}\left[D_{k}+\frac{\Phi_{k}}{4}\left(w_{i, j+1}^{n}-w_{i, j-1}^{n}\right)\right], \\
& \mathscr{P}_{3}: \mathscr{P}_{i, j-1}^{n}:=\frac{\Delta t}{(\Delta x)^{2}}\left[D_{k}-\frac{\Phi_{k}}{4}\left(w_{i, j+1}^{n}-w_{i, j-1}^{n}\right)\right], \\
& \mathscr{P}_{4}: \mathscr{P}_{i, j}^{n}:=1-4 D_{k} \frac{\Delta t}{(\Delta x)^{2}},
\end{aligned}
$$

where $k=\mathrm{E}, \mathrm{E} / \mathrm{M}, \mathrm{M}$ and, as throughout this paper, $0<D_{\mathrm{E}}<D_{\mathrm{E} / \mathrm{M}}<D_{\mathrm{M}}$ and $0=\Phi_{\mathrm{E}}<\Phi_{\mathrm{E} / \mathrm{M}}<\Phi_{\mathrm{M}}$. $\mathscr{P}_{0}, \mathscr{P}_{1}, \mathscr{P}_{2}, \mathscr{P}_{3}$ and $\mathscr{P}_{4}$ correspond to the probabilities that, during the next time step, a cancer cell at grid point $\left(x_{i}, y_{j}\right)$ moves left, right, up, down, and not at all, respectively (Anderson \& Chaplain, 1998; Anderson et al., 2000; Franssen et al., 2019). Note that if any of the coefficients $\mathscr{P}_{0}$ to $\mathscr{P}_{3}$ become negative, we set them to zero during that time step. Rules for proliferation and phenotypic transitions of the cancer cells (as well as - on the secondary grids - for cell death and dormancy) are then included at the discrete level, as described below.

The more proliferative cancer cells of epithelial phenotype perform mitosis after time interval $T_{\mathrm{E}}$ and the less proliferative partial-EMT and mesenchymal-like cancer cells after time interval $T_{\mathrm{E} / \mathrm{M}}$ and $T_{\mathrm{M}}$ (with $T_{\mathrm{E}} \leqslant T_{\mathrm{E} / \mathrm{M}} \leqslant T_{\mathrm{M}}$ ), respectively. As previously in Franssen et al. (2019), when proliferating, the cancer cells pass on their location so that a proliferating cancer cell is replaced by two daughter cells. Generally, during a proliferative step, cells are replaced by cells of their respective phenotype. However, in accordance with the biological findings presented in Section 2, the extended model allows for location-dependent full and partial EMT upon proliferation on the primary grid. As also explained schematically on the left-hand side of Figure 5, the EMT mutations on the primary grid occur as follows:

- Cancer cells of epithelial phenotype may be replaced by a set of daughter cells consisting of one cell of epithelial and one of partial-EMT phenotype with probability $\mathscr{P} \underset{\mathrm{EMT}}{\mathrm{E} / \mathrm{M}}>0$ when proliferating;

- If at least one neighbouring grid point of a cancer cell of epithelial phenotype is unoccupied, the cell may be replaced by a set of daughter cells consisting of one of epithelial and one of partial-EMT phenotype with an additional probability $\mathscr{P} \mathrm{EMT}$

- Cancer cells of epithelial and of partial-EMT phenotype may be replaced by a set of daughter cells consisting of one cell of epithelial or of partial-EMT phenotype, respectively, and one of mesenchymal phenotype with probability $\mathscr{P}_{\mathrm{EMT}}^{\mathrm{M}}<\mathscr{P}_{\mathrm{EMT}}^{\mathrm{E} / \mathrm{M}}$ when proliferating. 
As before, to account for competition for space and resources, the cancer cells on the respective grid point do not proliferate if there are already $\mathscr{Q} \in \mathbb{N}$ cancer cells on a grid point at the time of proliferation. Thus, $\mathscr{Q}$ represents the preferred carrying capacity in our model. If proliferation is not possible due to spatial constraints, the affected cell may proliferate again after another $T_{\mathrm{E}}, T_{\mathrm{E} / \mathrm{M}}$ or $T_{\mathrm{M}}$ time steps, respectively.

Intravasation As in Franssen et al. (2019), to represent the entry points into the blood vessels, a number of $U_{\mathrm{p}} \in \mathbb{N}_{0}$ normal blood vessels as well as $V_{\mathrm{p}} \in \mathbb{N}_{0}$ ruptured blood vessels are distributed throughout the primary grid. The normal blood vessels take the size of one grid point, while ruptured vessels consist of a group of $A^{b} \in \mathbb{N}$, where $b=1,2, \ldots, V_{\mathrm{P}}$, adjacent grid points and can thus have different shapes. The entry rules for cancer cells of epithelial and mesenchymal phenotype remain as described in Franssen et al. (2019). Moreover, in this extended framework, the cancer cells of partialEMT phenotype are treated in the same way as those of mesenchymal phenotype in the sense that they may intravasate into both ruptured vessels and-unlike epithelial-like cancer cells—normal vessels. Also, they are able to carry epithelial-like cancer cells into the vasculature with them.

Travel through the vasculature Cancer cells and cancer cell clusters remain in the vasculature for some time interval of length $T_{V} \in \mathbb{N}$, which biologically represents the average time the cancer cells spend in the blood system. If a cell would have normally been due to proliferate while in the vasculature, the proliferation is suppressed. It may proliferate again after another $T_{\mathrm{E}}, T_{\mathrm{E} / \mathrm{M}}$ or $T_{\mathrm{M}}$ time steps, as appropriate. Any cancer cells that enter a particular vessel at the same time are treated as one cluster and hence as a single entity once they are located in the vasculature. However, each cancer cell that is part of a cancer cell cluster disaggregates from its cluster with some probability $\mathscr{P}_{d}$ after spending a time interval of $\left[\frac{T_{V}}{2}\right\rceil$ in the vasculature. After the time interval $T_{V}$, the single cancer cells and the remaining cancer cell clusters are removed from the simulation unless they are randomly determined to survive. In accordance with the findings in Section 2, the survival probability is $\mathscr{P}_{\mathrm{E}}=\mathscr{P}_{\mathrm{M}}>0$ for single cancer cells of epithelial and mesenchymal phenotype, $\mathscr{P}_{\mathrm{E} / \mathrm{M}}>\mathscr{P}_{\mathrm{E}}, \mathscr{P}_{\mathrm{M}}$ for single cancer cells of partial-EMT phenotype, and $\mathscr{P}_{C}>\mathscr{P}_{\mathrm{E} / \mathrm{M}}$ for cancer cell clusters.

Metastatic growth On the secondary grids $\Omega_{\mathrm{S}}^{a}$, where $a=1,2, \ldots, G$, the same phenotypes of cancer cells are accounted for as on the primary grid. Also, the same movement probabilities from equations (3.3) are used to describe their movement. However, we allow for organ-specific adjustment of the cell movement through differentiation of the respective diffusion and haptotactic coefficients, $D_{k}^{\Omega_{\mathrm{S}}^{a}}$ and $\Phi_{k}^{\Omega_{\mathrm{S}}^{a}}, k=\mathrm{E}, \mathrm{E} / \mathrm{M}, \mathrm{M}, a=1,2, \ldots, G$.

Moreover, at the primary site, we model the assumption of well-adaptedness of the cancer cells to their tumour microenvironment of origin by considering proliferation every fixed time interval $T_{k}$, $k=\mathrm{E}, \mathrm{E} / \mathrm{M}, \mathrm{M}$, if the preferred carrying capacity $\mathscr{Q}$ permits. At the secondary sites, the cancer cells may not be as well-adapted to their new tumour microenvironment and may be exposed to the response of the immune system upon arrival. Furthermore, how well the cancer cells are adapted may vary between secondary organ tissues, $c f$. Section 2 . To account for this, we make several adjustments to the proliferative step on the secondary grids, which are summarised schematically on the right-hand side of Figure 5. Firstly, cancer cells may die with some grid-specific probability $\mathscr{P}_{D}^{\Omega_{S}^{a}}$ immediately prior to each potential proliferation. Secondly, a cell may not proliferate with some grid-specific probability $\mathscr{P}_{\delta}^{\Omega_{S}^{a}}$ when proliferation is due to account for cancer cell dormancy. Besides this, if cancer cells do proliferate during a time step, we account for MET at the secondary sites in accordance with the biological findings presented in Section 2. Hence, cancer cells of partial-EMT phenotype on the secondary 


\section{Mutations at primary site}
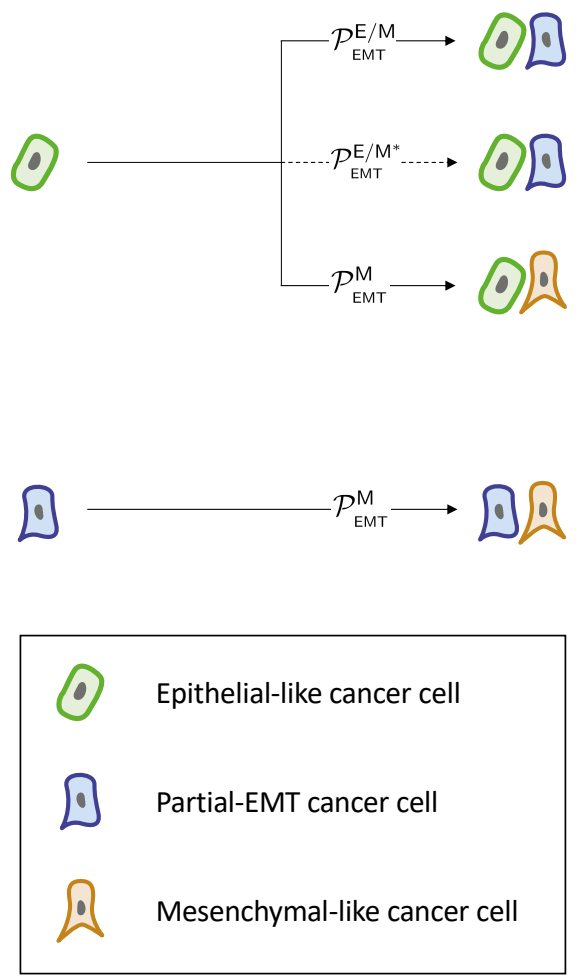

\section{Mutations, dormancy \& death at secondary sites}
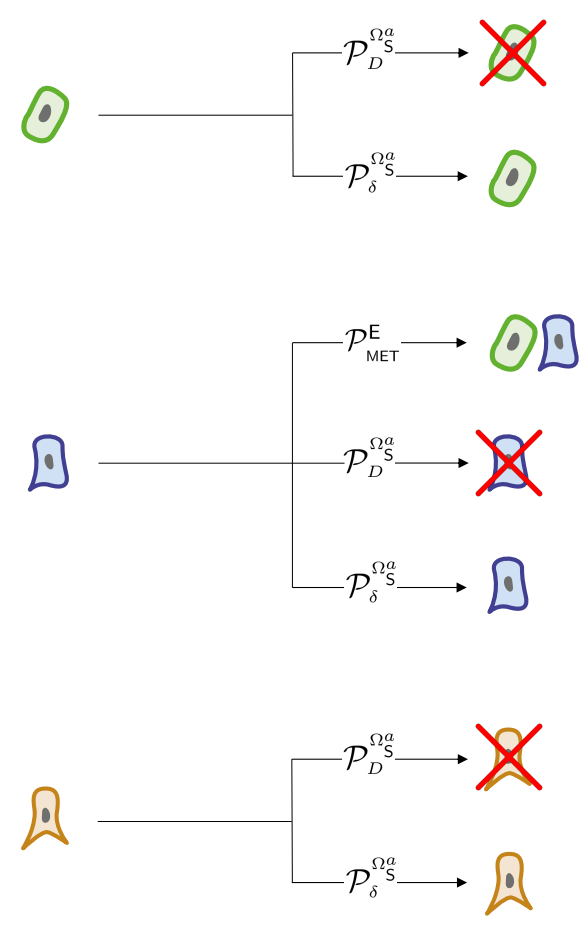

Figure 5. Schematic representation of possible EMT mutations at the primary site (left) and of MET mutations, cell death and dormancy at the secondary sites (right). Upon proliferation, each of the cells of the three phenotypes on the left of each arrow may undergo one of three fates instead of the usual proliferation: (A) It may be replaced by one cancer cell of the same and one of a different phenotype; (B) it may die (indicated by the red cross); or (C) it may remain dormant. (B) and (C) occur at secondary sites only (shown on the right). The dashed line on the top left indicates that this additional probability $\mathscr{P} \mathrm{E} / \mathrm{M}^{*}$ for cancer cells to mutate only applies to cancer cells at the tumour edge (see text). Note that, for enhanced readability, the illustration omits the representation of non-mutated proliferation, which results in the substitution of one parent cell by two daughter cells of its phenotype. Also, if the carrying capacity is reached on a grid point prior to proliferation, proliferation—and thus mutations—do not occur. 

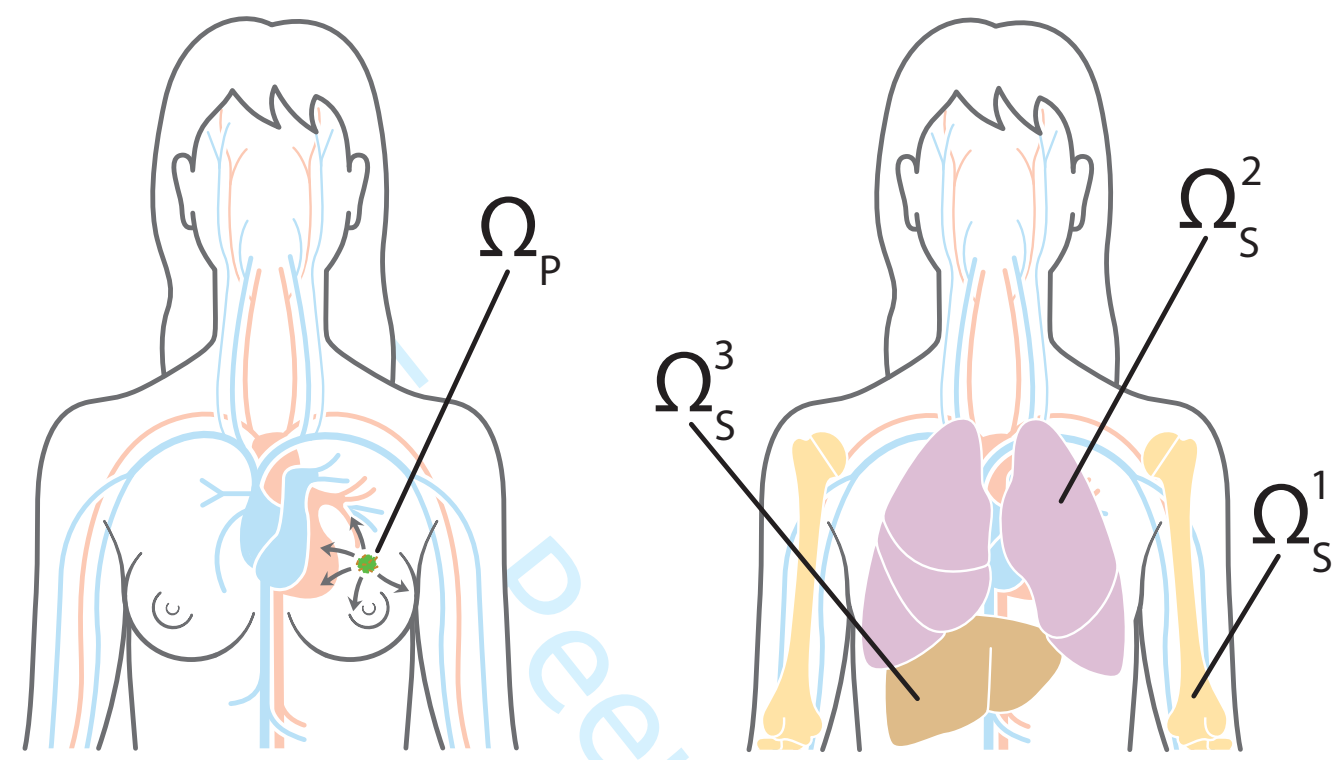

Figure 6. Primary and metastatic sites. To give an example of how the general modelling framework can be applied to a specific clinical setting, we chose the primary site $\Omega_{\mathrm{p}}$ in our simulations to represent the breast (left). Potential secondary metastatic sites $\Omega_{\mathrm{S}}^{1}, \Omega_{\mathrm{S}}^{2}, \Omega_{\mathrm{S}}^{3}$ were chosen to represent the bones, the lungs and the liver, respectively (right). Cancer cells can reach the secondary sites by travelling through the blood system.

grids may be replaced by a set of daughter cells consisting of one cancer cell of epithelial and one of partial-EMT phenotype with probability $\mathscr{P}_{\mathrm{MET}}^{\mathrm{E}}>0$ when proliferating. EMT does not occur on the secondary grids. However, as before, proliferation is capped as soon as a maximum of $\mathscr{Q}$ cancer cells per grid point is reached.

\section{Setup of computational simulations and model calibration}

To perform numerical simulations, we non-dimensionalised the system of equations (3.1)-(3.2) and the movement probabilities (3.3), with $k=\mathrm{E}, \mathrm{E} / \mathrm{M}, \mathrm{M}$, as described in Appendix A. In accordance with Anderson et al. (2000); Franssen et al. (2019), we chose to rescale distance with an appropriate length scale $L=0.2 \mathrm{~cm}$ (since $0.1-1 \mathrm{~cm}$ is estimated to be the maximum invasion distance of cancer cells at an early stage of cancer invasion) and time with an appropriate scaling parameter $\tau=\frac{L^{2}}{D}$. Here, $D=10^{-6} \mathrm{~cm}^{2} \mathrm{~s}^{-1}$ is a reference chemical diffusion coefficient suggested by Bray (1992), such that $\tau=4 \times 10^{4} \mathrm{~s}$, which corresponds to approximately $11 \mathrm{~h}$.

We considered spatial domains of size $[0,1] \times[0,1]$. This corresponds to physical domains of size $[0,0.2] \mathrm{cm} \times[0,0.2] \mathrm{cm}$. In particular, we let the spatial domain $\Omega_{\mathrm{p}}$ represent the primary site and the spatial domains $\Omega_{\mathrm{s}}^{1}, \Omega_{\mathrm{s}}^{2}$ and $\Omega_{\mathrm{s}}^{3}$ describe three potential metastatic sites. These spatial domains could represent any primary and secondary carcinoma sites. However, to give an example of a specific application, we chose $\Omega_{\mathrm{P}}$ to represent the primary site of the breast, and $\Omega_{\mathrm{S}}^{1}, \Omega_{\mathrm{S}}^{2}$ and $\Omega_{\mathrm{S}}^{3}$ to correspond to the bones, lungs and liver, respectively, which are commonly observed metastatic sites in breast cancer, $c f$. Figure 6.

The four spatial domains were discretised to contain $201 \times 201$ grid points each. This corresponds 
to a non-dimensionalised space step of $\Delta x=\Delta y=5 \times 10^{-3}$, which results in a dimensional space step of $1 \times 10^{-3} \mathrm{~cm}$, and thus roughly corresponds to the diameter of a breast cancer cell (Vajtai, 2013). We then chose a time step of $\Delta t=1 \times 10^{-3}$, corresponding to $40 \mathrm{~s}$. This ensures that the scheme complies with the Courant-Friedrichs-Lewy (CFL) condition (Anderson et al., 2000), while still maintaining appropriate computational efficiency.

We used an explicit forward-Euler in time, central-difference in space numerical scheme to solve the PDEs of our system. The time step was chosen to satisfy the von Neumann stability condition for the 2D diffusion equation (i.e. $\Delta t \leqslant \frac{h^{2}}{4 D}, h=\Delta x=\Delta y$ ), thus ensuring stability while maintaining appropriate computational efficiency and accuracy ( $c f$. Anderson \& Chaplain (1998); Anderson et al. (2000); Morton $\&$ Mayers (2005)). Specifically, the four spatial domains were discretised to contain $201 \times 201$ grid points each. This corresponds to a non-dimensionalised space step of $\Delta x=\Delta y=5 \times 10^{-3}$, which results in a dimensional space step of $1 \times 10^{-3} \mathrm{~cm}=10 \mu \mathrm{m}$, and thus roughly corresponds to the diameter of a breast cancer cell (Vajtai, 2013). We then chose a time step of $\Delta t=1 \times 10^{-3}$, corresponding to $40 \mathrm{~s}$. We ran the simulations for a time period corresponding to approximately 4 days.

On each secondary grid, we chose $U_{\mathrm{s}}^{1}=U_{\mathrm{s}}^{2}=U_{\mathrm{s}}^{3}=10$ distinct grid points, on which blood vessels are located. For each grid, these blood vessels were placed randomly but at least two grid step widths away from the respective grid's boundary. The same applies to the primary grid $\Omega_{\mathrm{P}}$ but with the additional condition that the $U_{\mathrm{P}}=8$ single grid points, where normal blood vessels are located, and the $V_{\mathrm{P}}=2$ sets of five grid points, where ruptured blood vessels are placed, are located outside a quasi-circular region containing the 200 centre-most grid points. While these 10 randomly placed vessels are modelled to exist from the beginning, they represent those vessels that grow as a result of tumour-induced angiogenesis in the vascular tumour growth phase-hence they are placed away from the initial avascular epithelial tumour mass.

To represent a two-dimensional cross-section of a small avascular primary tumour, we placed a nodule that consisted of 288 randomly distributed epithelial-like cancer cells in the quasi-circular region of the 97 centre-most grid points of the primary grid. To account for competition for space, we allowed for no more than $\mathscr{Q}=4$ cancer cells on any grid point. This preferred carrying capacity of $\mathscr{Q}=4$ was applied throughout the simulation. The described initial condition ensured that the cancer cells were placed away from any pre-existing vessels to match the biology of an avascular tumour in epithelial tissue. The counters for the cell age and proliferation were initially set to zero for all cancer cells. Figure 7 gives an example of a typical initial cancer cell placement and vessel distribution on the primary grid.

In accordance with the ranges provided in Table A.1, we set the epithelial-like cancer cell diffusion coefficient to $D_{\mathrm{E}}=1 \times 10^{-4}$, the partial-EMT cancer cell diffusion coefficient to $D_{\mathrm{E} / \mathrm{M}}=2.5 \times 10^{-4}$ and the mesenchymal-like cancer cell diffusion coefficient to $D_{\mathrm{M}}=5 \times 10^{-4}$. Furthermore, the epithelial, partial-EMT and mesenchymal haptotactic sensitivity coefficients were chosen to be $\Phi_{\mathrm{E}}=5 \times 10^{-5}$, $\Phi_{\mathrm{E} / \mathrm{M}}=1 \times 10^{-3}$, and $\Phi_{\mathrm{M}}=2 \times 10^{-3}$, respectively.

Taking into consideration the qualitative and quantitative biological findings in Section 2, we further assumed that, once in the vasculature, single CTCs of epithelial and mesenchymal phenotypes had a survival probability of $\mathscr{P}_{\mathrm{E}}=\mathscr{P}_{\mathrm{M}}=2 \times 10^{-4}$, while those of partial-EMT phenotype survived the travel through the vasculature with probability $\mathscr{P}_{\mathrm{E} / \mathrm{M}}=6 \times 10^{-4}$. The survival probability of CTC clusters was set to $\mathscr{P}_{C}=2.5 \times 10^{-2} \approx 42 \times \mathscr{P}_{\mathrm{E} / \mathrm{M}}$, in accordance with the finding by Aceto et al. (2014) that the survival probability of CTC clusters is between 23 and 50 times higher than that of single CTCs. Surviving single CTCs and CTC clusters exited onto the secondary grids after spending a time period of $T_{V}=0.18$ in the blood system, which corresponds to $2 \mathrm{~h}$ and hence to the breast cancer-specific clinical 


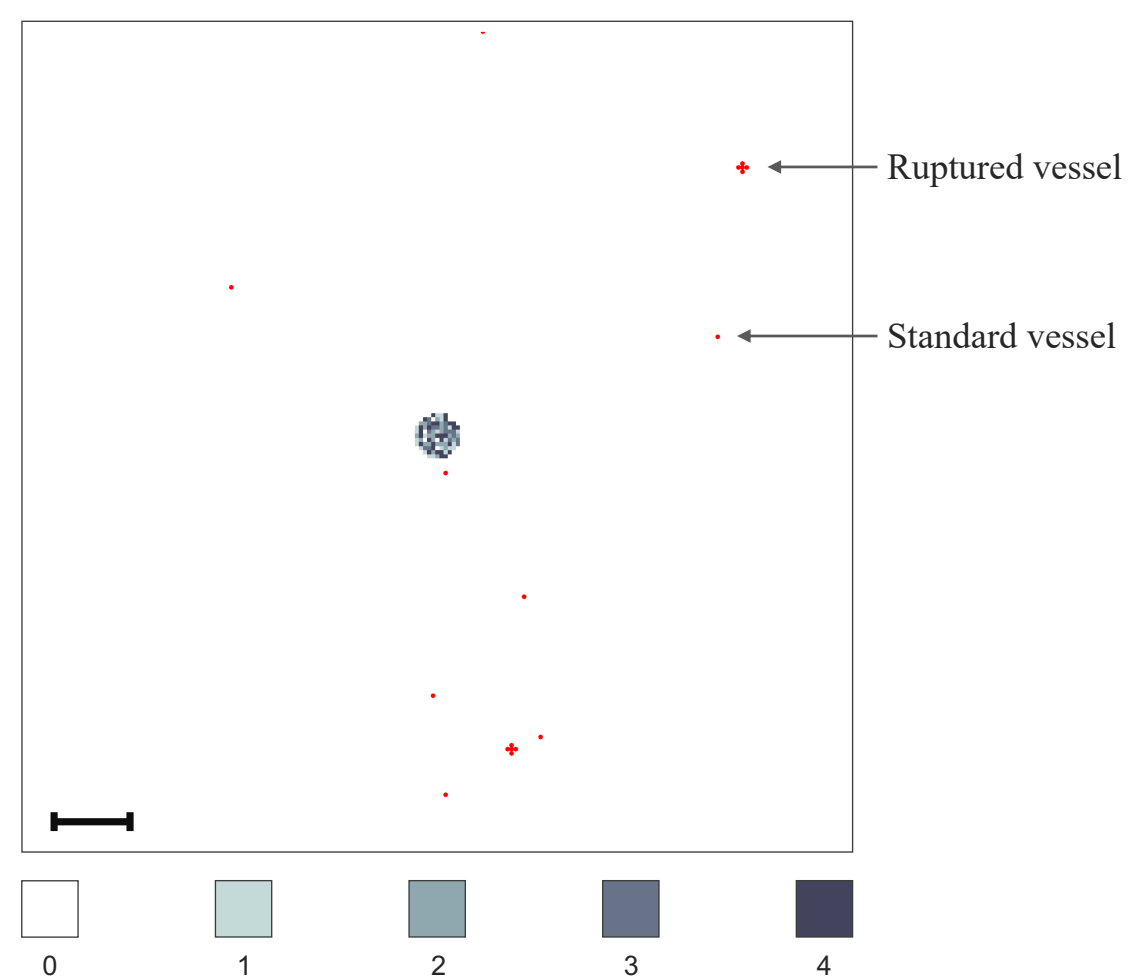

Figure 7. Vessel distribution and initial condition of cancer cells. The plot shows (in red) ten randomly distributed blood vessels on the primary grid, two of which are so-called ruptured vessels that consist of five rather than one grid point. In the centre of the grid, the initial distribution of epithelial-like cancer cells is shown. There are between 0 (white) and 4 (black) cancer cells on a grid point. As the initial distribution of cancer cells represents a 2D section through an avascular tumour, the blood vessels are placed at some distance away from the initial nodule of cancer cells. The scale bar denotes $0.02 \mathrm{~cm}$.

results in Meng et al. (2004).

Further, we assumed a uniform initial MMP-2 concentration of $m(0, \vec{x})=0$ across all the spatial domains. We varied the initial ECM density according to the organ each grid represents using clinical measurements of ECM densities in organs from ICRP (2009). These are presented in Table A.1. For this, we took $w(0, \vec{x})=1, \vec{x} \in \Omega_{\mathrm{P}}$, on the primary grid that represents the breast as our reference density. We then rescaled the initial ECM densities on the secondary grids relative to this initial density. For the bones, lungs and liver, respectively, this yielded $w(0, \vec{x}) \approx 0.9608$ for $\vec{x} \in \Omega_{\mathrm{s}}^{1}, w(0, \vec{x}) \approx 1.0392$ for $\vec{x} \in \Omega_{\mathrm{s}}^{2}$, and $w(0, \vec{x}) \approx 1.0294$ for $\vec{x} \in \Omega_{\mathrm{S}}^{3}$. We assumed that epithelial-like cancer cells divide by mitosis every interval $T_{\mathrm{E}}=2$, the partial-EMT cancer cells every interval $T_{\mathrm{E} / \mathrm{M}}=3$, and the mesenchymal-like cancer cells every $T_{\mathrm{E}}=6$. This corresponds to approximately 22 hours, 33 hours and 67 hours, respectively, which is consistent with the average doubling times found in breast cancer cell lines (Hughes et al., 2008; Milo et al., 2009; NCI, 2015). Moreover, we assumed that on the primary site, upon proliferation of a cancer cell of epithelial or partial-EMT phenotype, one of the daughter cells mutates into a mesenchymal-like cancer cell with probability $\mathscr{P}_{\mathrm{EMT}}^{\mathrm{M}}=1 \times 10^{-2}$. Similarly, one daughter cell of each epithelial-like cancer cell may mutate into a partial-EMT cancer cell with probability $\mathscr{P} \mathrm{E} / \mathrm{M}=2 \times 10^{-2}$ throughout the grid. 
Moreover, there is an additional probability for partial EMT of $\mathscr{P} \mathrm{EMT}^{\mathrm{E}} \mathrm{M}^{*}=0.15$ if the epithelial-like cancer cell is located at the edge of the tumour. Instead of representing the adaptation to each grid through these parameter settings, we determined the relative likelihood of metastasis-formation at the three secondary sites by consulting data on the transition probabilities of primary breast cancer to the metastatic sites of the bones, lungs and liver, respectively. As in Franssen et al. (2019), we used data gathered in a study of 4181 breast cancer patients (Kuhn Laboratory, 2017). As shown in Figure 4 of Franssen et al. (2019), the one-step transition probability from the breast to the bones was $23.1 \%$, to the lungs was $15.3 \%$ and to the liver was $11.0 \%$. Since we focus solely on the spread to these three metastatic sites and the spread to other organs is included in the terms accounting for vascular death, we obtain the relative likelihoods of spread to the bones, lungs and liver, which are $\mathscr{P}_{\mathrm{S}}^{1} \approx 0.5461, \mathscr{P}_{\mathrm{S}}^{2} \approx 0.2553$ and $\mathscr{P}_{\mathrm{s}}^{3} \approx 0.1986$, respectively.

At the secondary sites, cancer cells of partial-EMT phenotype were replaced by a set of daughter cells, consisting of one cell of epithelial and one of partial-EMT phenotype, with probability $\mathscr{P} \mathrm{MET}$ during proliferation. Due to a lack of organ-specific data on differences in the tumour microenvironments that could affect the diffusion and haptotactic coefficients of the cancer cells of various phenotypes - as well as their dormancy and death probabilities - at the time of writing, we restricted the differentiation between organs to their local initial ECM density at this stage. Accordingly, we-as for the primary grid-set $D_{k}^{\Omega_{\mathrm{S}}^{a}}=D_{k}$ and $\Phi_{k}^{\Omega_{\mathrm{S}}^{a}}=\Phi_{k}$, where $k=\mathrm{E}, \mathrm{E} / \mathrm{M}, \mathrm{M}$ and for $a=1,2,3$, on all secondary grids in the model. Similarly, the dormancy and death probabilities on all secondary grids were $\mathscr{P}_{\delta}^{\Omega_{S}^{a}}=0.5$ and $\mathscr{P}_{D}^{\Omega_{S}^{a}}=0.05, a=1,2,3$. An overview of the parameter values mentioned herein can be found in Table A.1.

\section{Computational simulation results}

To verify that the modelling framework is able to capture the key steps of the invasion-metastasis cascade, we ran simulations with the parameters shown in Table A.1. All simulations were run on a standard desktop computer. The average computational run time for a simulation was approximately 45 minutes. We provide sample results showing the primary and the three secondary grids at various times in the range of 0 to 24 days during one sample simulation in Figure 8 and Figures 9-11, respectively. We chose results on the primary grid to show the spatiotemporal dynamics on day 0,11 and 22 so that they can be contrasted to those in Franssen et al. (2019). For the secondary sites, we chose to present sample results for times that best give evidence of the various mechanisms related to metastatic spread, MET and the consequences of the immune response at secondary sites that are described through this modelling framework. However, this does not imply that these phenomena are limited to the times and locations depicted in Figures 8-11 in that particular or in other simulations.

As described in Section 4, we started the simulations with a small nodule of epithelial-like cancer cells of diameter $\sim 1.5 \times 10^{-2} \mathrm{~cm}$ ( $c f$. Figure 7). These were located on the primary grid representing the breast, which had an ECM of uniform density and contained no partial-EMT cancer cells, no mesenchymal-like cancer cells and no MMP-2, as shown in the left-most column of Figure 8. As the middle column of Figure 8 shows, after 11 days, the epithelial-like cancer cells had invaded the local tissue, covering a nearly circular area of approximately $0.1 \mathrm{~cm}$ diameter. Moreover, some partial-EMT and mesenchymal-like cancer cells could be observed on the primary grid. Their occurrence arose from cancer cells of previously epithelial-like phenotype via (partial) EMT. Both of these cell types occurred sparsely within a quasi-circular region with an approximate diameter of $0.18 \mathrm{~cm}$. Additionally, the 


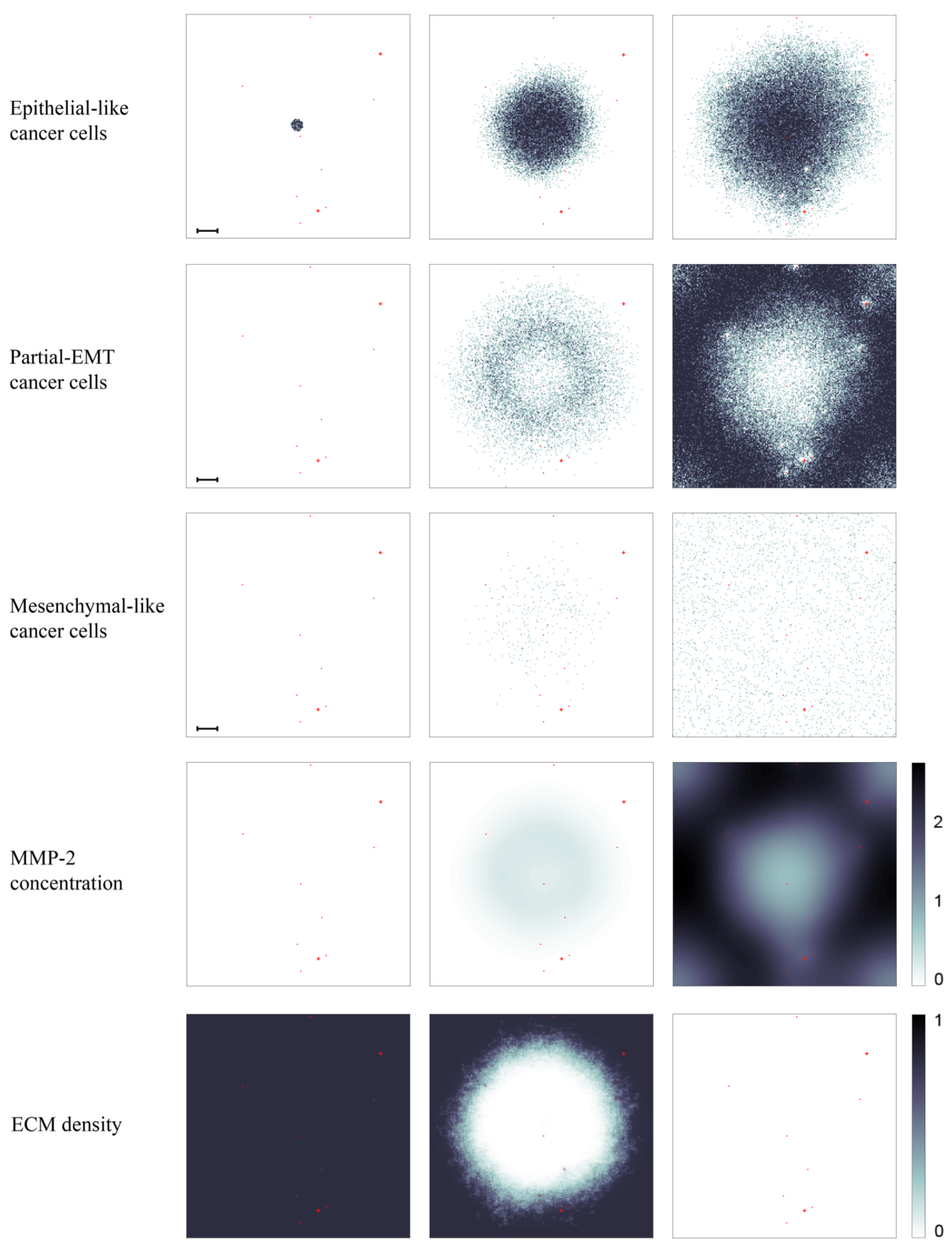

Figure 8 . Simulation results on the primary grid. Primary tumour dynamics at 0 days, $\sim 11$ days and $\sim 22$ days. For each time step, the distribution of epithelial-like, partial-EMT and mesenchymal-like cancer cells (first to third row) is shown, with the discrete number of cancer cells per grid point ranging from 0 (white) to 4 (black) on each of the panels. The MMP- 2 concentration (fourth row) continuously varies between 0 (white) and 2.6602 (black), and the ECM density (bottom row) between 0 and 1 . Red dots represent blood vessels. There are 8 normal blood vessels of the size of one grid point as well as 2 ruptured blood vessels, which extend over 5 grid points each. If cancer cells are moved to these grid points, they may enter the vasculature and can potentially extravasate at a secondary site ( $c f$. Figures 9-11). The scale bar denotes $0.02 \mathrm{~cm}$ and applies to all of the panels. 
21.1 days

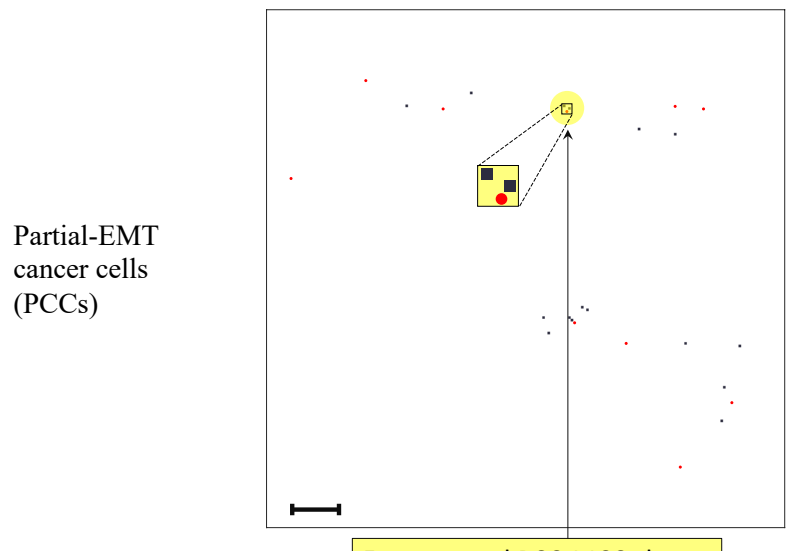

Extravasated PCC-MCC cluster

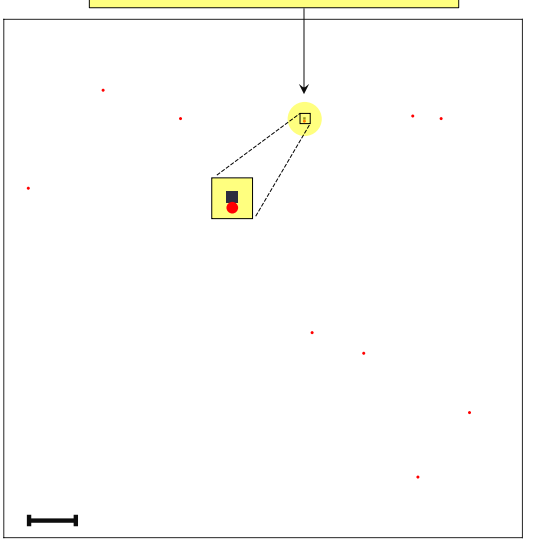

Mesenchymal-like cancer cells (MCCs)
24.1 days

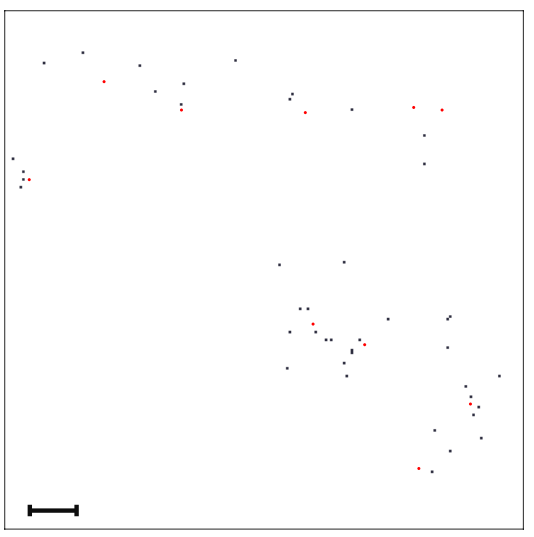

Dormant MCC

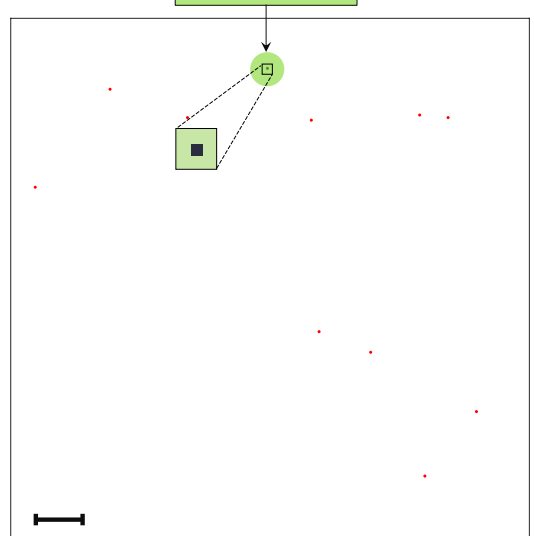

Figure 9. Cluster extravasation and dormancy on secondary grid representing the bones. Distribution of partial-EMT cancer cells (upper panels) and mesenchymal-like cancer cells (lower panels) at the secondary site representing the bones is shown after $\sim 21$ days (left) and $\sim 24$ days (right). The number of cancer cells per grid point varies between 0 (white) and 1 (black). Around day 21, a cluster consisting of two partial-EMT and one mesenchymal-like cancer cell extravasates onto the grid of the bones (yellow). Moreover, over the 3 day period between the panels on the left and on the right, the mesenchymal-like cancer cell, which normally has a doubling-time of $\sim 2.78$ days, remains dormant (green). The scale bar denotes $0.02 \mathrm{~cm}$. 
16.3 days

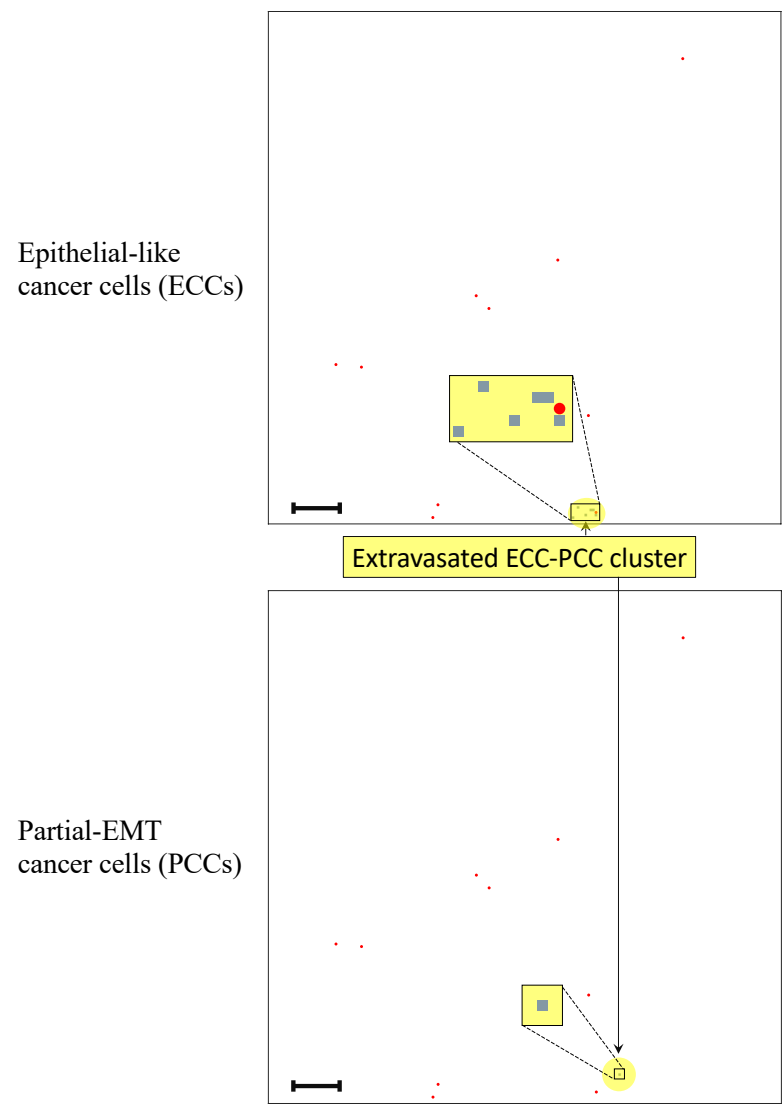

23.1 days
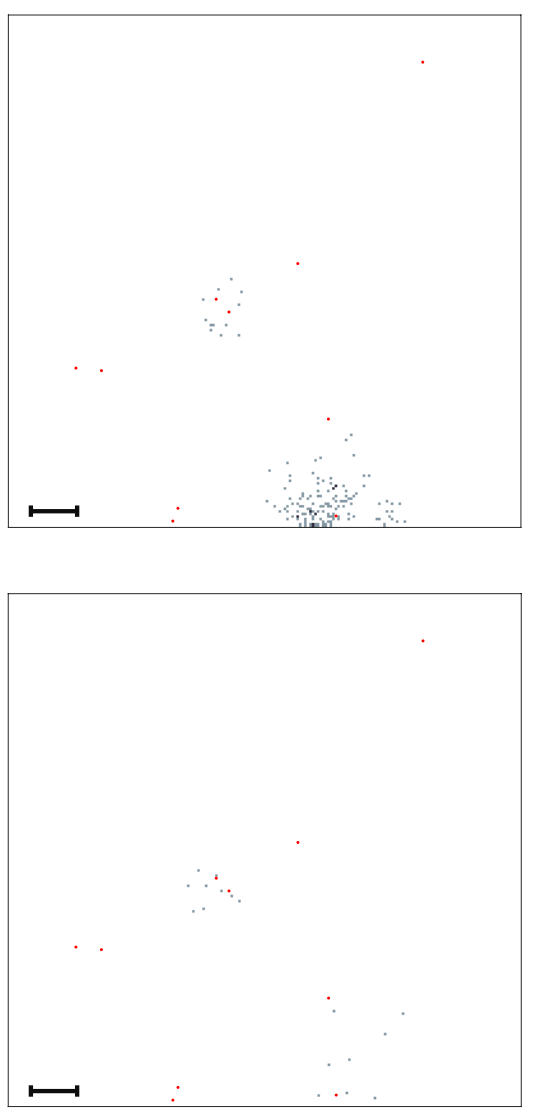

Figure 10. Cluster extravasation and largest metastatic lesion on secondary grid representing the lungs. Distribution of epithelial-like cancer cells (upper panels) and partial-EMT cancer cells (lower panels) at the secondary site representing the lungs is shown after $\sim 16$ days (left) and $\sim 23$ days (right). The number of cancer cells per grid point varies between 0 (white) and 2 (black). Around day 16, a cluster consisting of six epithelial-like and one partial-EMT cancer cell extravasates onto the grid of the lungs (yellow). This early extravasation of a relatively large cluster of epithelial-like cancer cells results in the largest metastatic growth that can be observed during the time period of this simulation in the panels on the right. The scale bar denotes $0.02 \mathrm{~cm}$. 

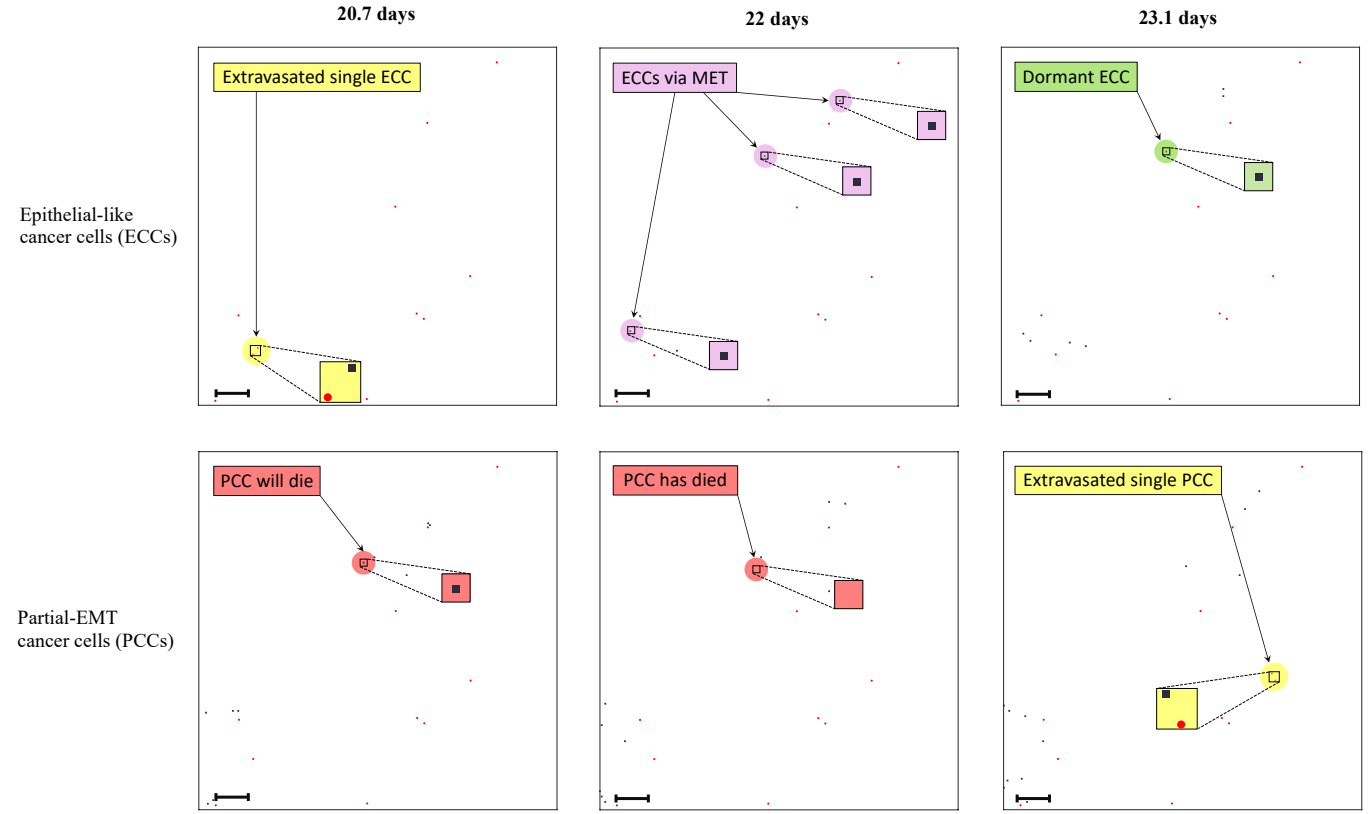

Figure 11. Single cell extravasations, MET, dormancy and cell death on secondary grid representing the liver. Distribution of epithelial-like cancer cells (upper panels) and partial-EMT cancer cells (lower panels) at the secondary site representing the liver is shown after $\sim 21$ days, $\sim 22$ days and $\sim 23$ days (left to right). The number of cancer cells per grid point varies between 0 (white) and 1 (black). On day 20 and 23, a single epithelial-like and a single partial-EMT cancer cell extravasate onto the grid of the liver (yellow). No extravasations took place during the presented time period. Hence, the three epithelial-like cancer cells that occurred in the period between 20.7 days and 22 days in the upper middle panel (pink) are a result of MET of the partial-EMT cancer cells presented in the bottom row of panels. During the same time period, a partial-EMT cancer cell dies (red). Moreover, over the 1.1 day period between the panels in the middle and on the right, an epithelial-like cancer cell, which normally has a doubling-time of $\sim 0.93$ days, remains dormant (green). The scale bar denotes $0.02 \mathrm{~cm}$.

partial-EMT cancer cells populated a ring-shaped area at the edge of the tumour more densely. The MMP-2 concentration broadly followed the distribution of the partial-EMT cells and ranged from 0 to 0.38. Moreover, the ECM had been degraded in the centre of the grid and a density gradient could be observed at the edge of this near-circular region. After 22 days, the area occupied by the epitheliallike cancer cells in the centre of the tumour had expanded further. Also, the ring-like area populated with partial-EMT cancer cells at the tumour edge had grown and become more densely populated. The mesenchymal-like cancer cells were now sparsely spread throughout the whole grid. In general, we observed that areas on the grid near vessels were sparsely occupied, if at all. The distribution of the MMP-2 concentration still broadly followed the evolution of the partial-EMT cancer cells, now ranging from 0.76 to 2.66. The ECM on the domain that we considered had now been fully degraded.

In addition to the cancer cell invasion on the primary grid, we also observed metastatic spread to the grids representing the secondary sites. In Franssen et al. (2019), we showed all of the secondary grids at the same time instances as the primary grid, so after approximately 11 and 22 days. Moreover, we included the spatiotemporal dynamics of the ECM density and of the MMP-2 concentration for each of the grids at these times. For these results, we hence refer the interested reader to this previous work. In 

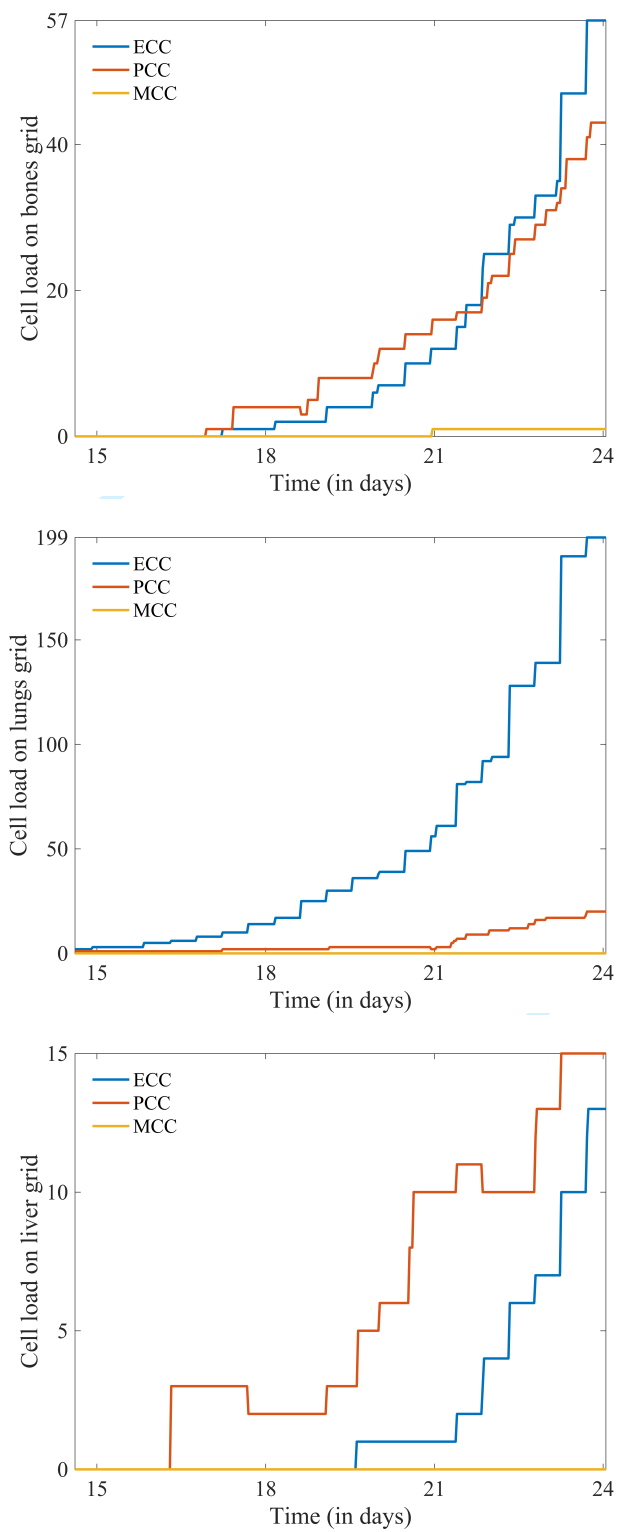

Figure 12. Phenotype-specific cell load over time on secondary grids. Plots of total number of epithelial-like (ECC; blue), partial-EMT (PCC; red) and mesenchymal-like (MCC; yellow) cancer cells on the grids of the bones, lungs and liver (top to bottom) in the period between 14.6 and 24.1 days. On each grid, the initial growth arises from an extravasation of cells. The stepwise, mostly non-negative growth pattern thereafter largely occurs from—often synchronous within the phenotypic groupproliferation of cells in combination with further extravasations. For ECCs, part of the growth also results from PCCs that undergo MET. As MET during PCC proliferation results in one PCC and one ECC, MET typically causes the PCC growth to slow down. Negative growth, as e.g. observed in the PCC population on the top 'bones' grid after day 18, on the middle 'lungs' grid at day 21 , and on the bottom 'liver' grid after day 17 and 22, is always a result of cell death. Throughout, MCCs on the secondary grids remain rare. 

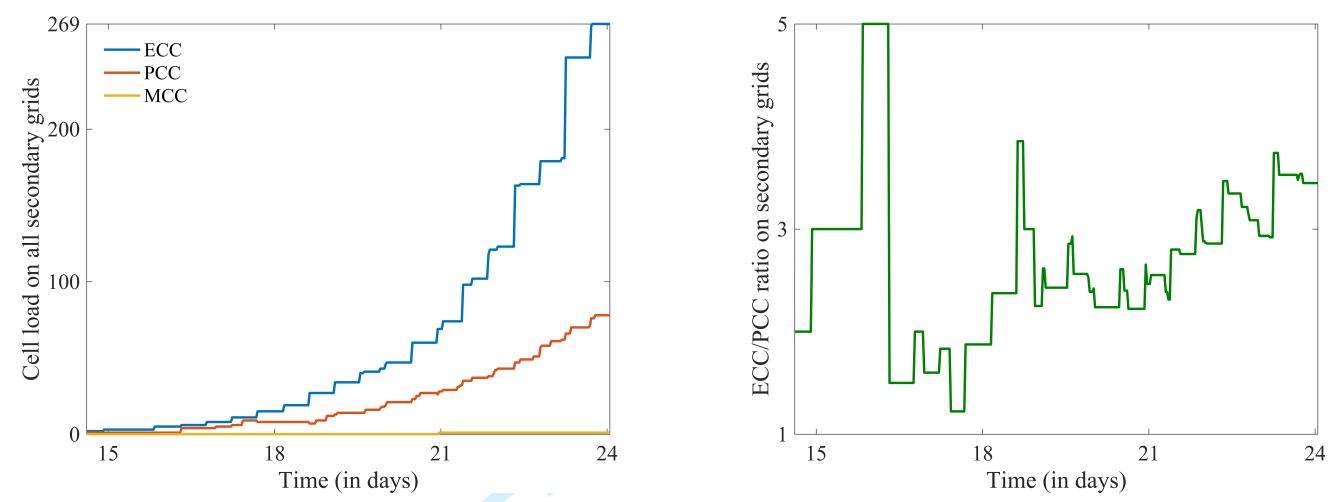

Figure 13. Trends in phenotype-specific cell load on secondary grids overall. Left: Combined total cell load on the secondary grids of cancer cells of epithelial-like (ECC; blue), partial-EMT (PCC; red) and mesenchymal-like (MCC; yellow) phenotype between the period of 14.6 and 24.1 days. The number of ECCs grows most rapidly over time-their growth is caused by extravasations, MET and proliferation. PCCs grow steadily but less rapidly. Their growth is slower due to their larger proliferation interval but also due to a subset of PCCs undergoing MET during proliferation. Only $1 \mathrm{MCC}$ is observed over the time period. Right: Plot of the ratio of ECCs to PCCs over the same time period (green). Throughout, there are more ECCs than PCCs.

this paper, we focus on the presentation of the additional phenomena captured through the extension of the model instead. As the newly introduced features are connected to the cells of various phenotypes that are included in the model, at the secondary sites we only show their evolution, while omitting the presentation of the MMP-2 and ECM dynamics. To give examples of how the various mechanisms that this model describes are instantiated in the simulations, we show the grids of the bones (Figure 9), the lungs (Figure 10) and the liver (Figure 11) at various times ranging from 16.3 to 24.1 days. The particular times differ between the secondary grids as they were chosen in order to best present how the phenomena occur in the simulations. Yet, within each grid, the time instances shown are such that the cell phenotypes depicted in the corresponding panels are at least the length of a cell doubling interval apart to allow all cells in the respective grid to have proliferated, if applicable, at least once.

We proceed by describing the results at the secondary sites grouped by the mechanisms that we aim to highlight (Figures 9-11) rather than grid-by-grid, as these mechanisms typically occur on all secondary grids. Furthermore, we present the dynamics of the cell-phenotype evolution of the population sizes on the secondary grids in Figures 12 and 13. Finally, we show trends in phenotype-specific cell load on the secondary grids overall in Figure 14.

\section{Extravasations}

We observed extravasations of single cancer cells of various phenotypes, as well as of homogeneous and heterogeneous cancer cell clusters. Examples of a selection of these extravasations are highlighted in yellow on the grids representing the various secondary organs. Figure 11 shows samples of recently extravasated single cancer cells of epithelial and of partial-EMT phenotype on the grid representing the liver. Figures 9 and 10 show examples of extravasated cancer cell clusters consisting of mixed phenotypes. These consist of two partial-EMT and one mesenchymal-like cancer cell on the grid that represents the bones, and of six epithelial-like and one partial-EMT cancer cell in the case of the grid representing the lungs. 

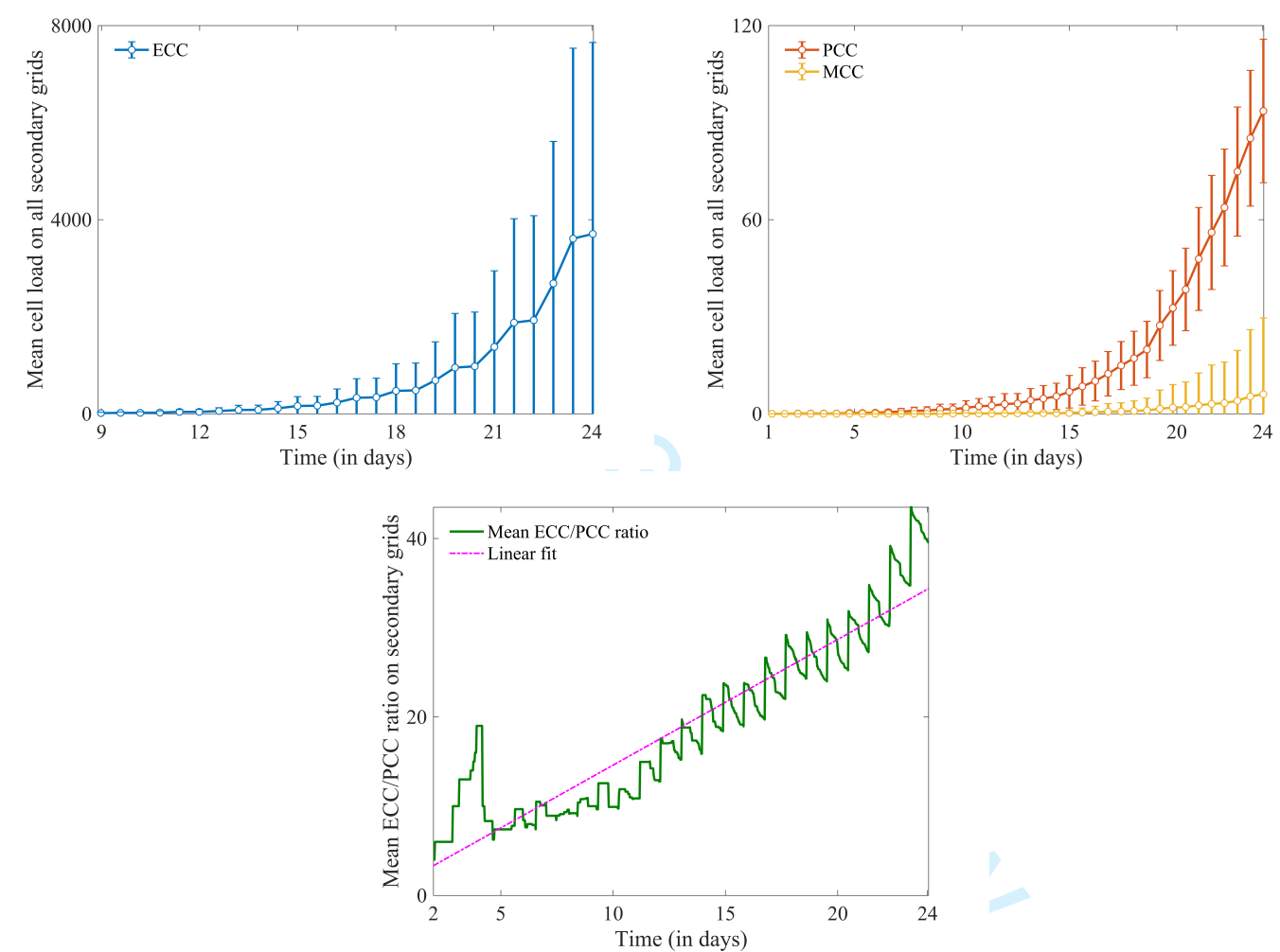

Figure 14. Trends in phenotype-specific cell load on secondary grids overall—showing the mean (line) and standard derivation (whiskers) from 20 simulations. All 20 simulations were run with the same conditions as the sample simulation in Figure 13 apart from that the 288 initial epithelial-like cancer cells were newly distributed randomly in the central 97 grid points and that different seeds for the random number generator were used for each simulation. Top left: Mean combined total load of cancer cells of epithelial-like (ECC; blue) phenotype on the secondary grids between the period of 9 and 24.1 days. The number of ECCs grows most rapidly over time - their growth is caused by extravasations, MET and proliferation. Top right: Mean combined total load of cancer cells of partial-EMT (PCC; red) and mesenchymal-like (MCC; yellow) phenotype on the secondary grids between the period of 1 and 24.1 days. Generally, PCCs grow steadily but less rapidly than ECCs. Their growth is slower due to their longer proliferation interval but also because a subset of PCCs undergoes MET during proliferation. MCCs also tend to grow in number over time, yet very slowly. This is due to their long doubling time. Bottom: Plot of the mean ratio of ECCs to PCCs between the period of 2 and 24.1 days (green). Throughout, there are on average more ECCs than PCCs and the mean ratio increases with time. The best linear fit line (pink) highlights this trend. 
During the 22 day period over which the simulation on the primary grid was run, we observed 6 extravasations of single cells, as well as 11 extravasations by clusters consisting of two cells, 6 by clusters consisting of three cells and 1 extravasation each by clusters consisting of six and of seven cells. Another general observation was that during the simulated 24 day period, only one mesenchymal-like cancer cell successfully extravasated onto a secondary grid. All other extravasations were performed by single cancer cells as well as by homo- and heterogeneous cancer cell clusters, which were mainly of partial-EMT phenotype but also of epithelial-like phenotype. The highest number of extravasations of either a cancer cell or a cancer cell cluster was observed onto the grid of the bones.

We ran an additional 19 simulations with the same conditions-including the vessel locations on all grids-as the sample simulation, apart from that the 288 initial epithelial-like cancer cells were newly distributed randomly in the central 97 grid points each time. Also, different seeds for the random number generator were used for each simulation, which affects various processes such as the movement and mutations of the cancer cells. Taking into consideration these 20 simulations, we observed the earliest extravasations to secondary grids by mesenchymal-like cancer cells after 9.3 days, by partialEMT cancer cells after 2 days and by epithelial-like cancer cells after 1.4 days, $c f$. Figure 14 . In other simulations, there were no extravasations by epithelial-like or mesenchymal-like cancer cells during the 24.1 days that we ran the simulations for.

\section{MET}

On the grid representing the liver, no extravasations took place during the time period between 20.7 and 23.1 days, i.e. during the period shown in Figure 11. Hence, the three epithelial-like cancer cells that occurred during the period between 20.7 and 22 days, which are highlighted in pink in the upper row of panels, are a result of MET of the partial-EMT cancer cells presented in the bottom row of panels. If MET occurred during a proliferative step, the respective partial-EMT cancer cell was replaced by one cancer cell of its own phenotype as well as one of epithelial-like phenotype. Overall, the phenomenon of MET caused the growth rate of epithelial-like cancer cells to increase while slowing the growth rate of partial-EMT cancer cells at secondary sites. This trend is captured in the plots in Figure 13.

While any partial-EMT cancer cell can potentially undergo MET, the mesenchymal-like cancer cells cannot change phenotype. The sole mesenchymal-like cancer cell in Figure 9 is an example of such a phenotypically stable cell.

\section{Metastatic growth}

The by far largest micrometastatic lesion during the simulation period presented itself on the grid of the lungs, shown in Figure 10. It resulted from a cluster consisting of six epithelial-like cancer cells and one partial-EMT cancer cell that extravasated relatively early-after approximately 16 days. All other lesions remained comparatively small during the same 23 day period, consisting of less than 20 cancer cells of almost exclusively epithelial-like and partial-EMT phenotypes. This is also reflected in the evolution of the total cell number on the three grids represented through the plots in Figure 12. As time progressed, a tendency towards a higher percentage of epithelial-like cancer cells at secondary sites was observed, as Figure 13 shows.

In the 20 simulations that we ran to examine the mean numbers of cells of the various phenotypes on secondary grids, $c f$. Figure 14, we observed a comparatively large standard deviation with respect to the mean number of epithelial-like cancer cells on the secondary grids. This is likely caused by the large variation in the time of the first extravasation by epithelial-like cancer cells, which occurred after as 
little as 1.4 days during one of the 20 simulations, while no epithelial-like cancer cells at all extravasated during another. Due to the exponential growth observed in the early stages of secondary spread when space is not yet a significantly limiting factor, large metastases can form if the extravasation occurred early on. The same applies to a lesser extent to the cells of the other two phenotypes. The standard deviation for the number of cells on the secondary grids over time is lower for these cell types. One reason is that cells of these phenotypes need to come into existence via mutations on the primary grid before they are able to spread. Also, they are less proliferative and, in the case of partial-EMT cancer cells, subject to MET on the secondary grids.

\section{Dormancy}

Given that we have chosen the periods between the time instances presented through the panels in Figures 9 to 11 to be such that there exists at least one opportunity for each cancer cell to reproduce, these figures show examples of dormant cancer cells at the secondary site of the bones and the liver, respectively. Due to their dormancy, the respective mesenchymal-like and epithelial-like cancer cells do not proliferate while other cells on the grids may. The two examples of dormant cells discussed in this section are highlighted in green in the respective Figures 9 and 11.

\section{Death due to maladaptation $\&$ immune response}

Figure 11 shows an example of a partial-EMT cancer cell that dies in the period between 20.7 and 22 days. Other examples of cell death on secondary sites become evident when examining the cell population growth plots for the partial-EMT cells on each of the secondary sites shown in Figure 12. Negative growth, as e.g. observed in the partial-EMT population on the top 'bones' grid after day 18, on the middle 'lungs' grid at day 21, and on the bottom 'liver' grid after day 17 and 22 , is always a result of cell death. The fact that we only observe cell death in the partial-EMT population through these plots does not imply that cell death does cannot occur in the cell populations of other phenotypes. The epithelial-like cancer cells in the model tend to proliferate mostly synchronously. Hence, rare potential cell deaths are likely to be overshadowed in the plots in Figure 12 by an even larger positive cell growth at the same time instance. The same applies to potential other partial-EMT cell deaths.

\section{Discussion and perspective}

In this paper, we have extended the mathematical framework for the metastatic spread of cancer originally proposed in Franssen et al. (2019) to include EMT and MET. As a result, the framework now additionally accounts for transitions of cancer cells between an epithelial, a newly introduced partialEMT and a mesenchymal phenotypic state. This is achieved in a location-dependent fashion-both with respect to the steps of the invasion-metastasis cascade and with respect to the intra-tumoural location of the cancer cells. This way, the modelling framework captures the phenomena of EMT and MET in their physiological context. Furthermore, we include organ-specific differences in the local tissue of the secondary sites involved in our model by accounting for their ECM density in accordance with biological findings in ICRP (2009). Finally, the extended framework now also takes into account cancer cell dormancy and death as a result of maladaptation to the new tumour microenvironments at the secondary sites as well as due to the local immune response.

As mentioned in Section 1, over $90 \%$ of cancer-related deaths are the result of metastatic spread rather than the primary tumour's growth alone. A predictive framework of the metastatic process has the potential to improve clinical outcomes. If a primary tumour is detected at an early stage, a predictive tool 
like the one presented here can simulate whether or not a primary tumour is likely to metastasise and, if so, over which timescale and to which secondary location(s). This consequently allows clinicians to use adjuvant therapy at appropriate secondary sites even before metastatic spread becomes clinically visible. Similarly, if cancer is detected at a late stage either at the primary or a secondary site, likely other sites of spread can be inferred. The most probable sites for cancerous growth can then be investigated in the clinic and treated appropriately. Here, the short running time of our model is a further advantage since time-expressed through disease progression—heavily impacts the treatment success. Furthermore, factors accounted for in the simulations such as the initial phenotypic detail, dimensions and shape of a carcinoma can be determined for each patient individually. This provides individualised initial conditions and thus simulation results. To ensure an accurate representation of the mechanistic process underlying the invasion-metastasis cascade, models of metastatic spread should account for EMT and MET. With regards to the effort of developing a predictive in silico tool, another compelling reason to include EMT processes is the major contribution of EMT to treatment resistance (Roche, 2018).

Through computational simulations, we found that the extended metastasis modelling framework provides biologically realistic outcomes and gives further insight into the above-described mechanisms that underpin the invasion-metastasis cascade at the cellular scale. Tumour shape and metastatic distribution at the primary site were predicted to appear as one would expect in a cancer patient who has not yet received treatment. In particular, we found that the partial-EMT cancer cells formed a ring-shaped leading front along the tumour edge, which was also seen in experiments (Nurmenniemi et al., 2009) as well as in human tissue, as shown in Figure 3 from Puram et al. (2017).

Nurmenniemi et al. (2009) further observed an average maximum invasion depth-measured as the distance from the centre of the grid to the cell furthest away from the centre-of $5.47 \times 10^{-2} \mathrm{~cm}$ over 14 days when culturing HSC-3 cancer cells, a human oral squamous carcinoma cell line with high metastatic potential, on top of myoma tissue. This translates into an average maximum invasion speed of approximately $4.52 \times 10^{-8} \mathrm{~cm} \mathrm{~s}^{-1}$. It suggests that our observed maximum invasion depth of $\sim 9 \times 10^{-2} \mathrm{~cm}$ in 11 days by partial-EMT and mesenchymal-like cancer cells, which remained roughly the same during the 20 simulations that we ran, and the resulting estimated average for the maximum invasion speed of approximately $9.38 \times 10^{-8} \mathrm{~cm} \mathrm{~s}^{-1}$ are realistic results-especially if we take into account that migration speed varies between cancer cell lines and that the displacement of the cancer cells in the simulation is likely a result of a combination of migration and proliferation.

To our knowledge, there are currently no data available that claim to deliver an accurate estimation of the typical metastatic load from primary breast cancer to secondary sites over a specified time frame. However, we believe that our results are biologically appropriate with regards to their timings. They are in correspondence with the conclusion reached by Obenauf \& Massague (2015) in their review of the metastatic traits that allow cancer cells to colonise various secondary sites, suggesting that CTCs and metastatic spread can be detected soon after vascularisation of the primary tumour, as in our simulations.

The types of extravasations that we observed through the simulations of our model coincide with the biological evidence presented in Section 2 that CTCs of all phenotypes appear to be able to extravasate (Banyard \& Bielenberg, 2015). Furthermore, only a low proportion of extravasations included mesenchymal-like cancer cells - the bulk of extravasating cells were of partial-EMT phenotype and others of epithelial phenotype.

The highest number of extravasations was observed onto the grid representing the bones. Yet, as Figure 10 indicates, the largest micrometastasis, which resulted from the early metastatic spread of a large cluster consisting predominantly of epithelial-like cancer cells, occurred at the site of the lungs, where only two extravasations were observed over the total time period that we considered. This emphasises that cancerous spread is highly complex and difficult to predict, a feature represented through 
the stochasticity involved in multiple processes of our model. Examples of such processes are (partial) EMT at the primary site, the survival of CTCs and the potential partial or full dissemination of CTC clusters in the vasculature, the determination of the secondary site of extravasation, as well as MET, dormancy and cell death at secondary sites. Furthermore, the fact that the largest growth stemmed from a cluster consisting of predominantly epithelial-like cancer cells highlights that this cell type with its distinguishing feature of rapid proliferation is generally the one best adapted to growth in the tumour microenvironment at secondary sites. This observation and our observation that-as time progressesincreasing numbers of partial-EMT cancer cells transit to an epithelial-like phenotype, $c f$. Figure 14, coincide with two of the biological findings discussed in Section 2. The first such finding is that the bulk of cancer cells at secondary sites are of epithelial-like phenotype (Pastushenko \& Blanpain, 2018), as well as some of partial-EMT phenotype (Dongre \& Weinberg, 2019). The second finding in agreement with our results is the observation by Ruscetti et al. (2015) that macrometastases at the secondary site of the lungs consisted mainly of epithelial-like cancer cells while smaller lesions presented few epithelial-like cancer cells and thus mainly cells with some degree of mesenchymal-traits. Finally, our model accounts for the biological evidence presented in Ocaña et al. (2012); Kröger et al. (2019) that cancer cells of a stable mesenchymal-like phenotype are unable to transform via MET and hence fail to give rise to metastatic growth at secondary sites.

In the current modelling approach, we account for the fact that EMT and MET have been observed to occur in specific steps of the invasion-metastasis cascade as well as in specific locations within the primary tumour. For instance, partial EMT appears to be triggered predominantly at the primary site and towards the tumour boundary, as observed in situ-see Figure 3 and Puram et al. (2017). Also, in the early stages of colonisation at a secondary site, MET is the predominant mutation. It would be desirable to additionally include a physiological motivation for the mutations that we model, like e.g. developed in Sfakianakis et al. (2017). In particular, we aim to incorporate the physiological motivation by accounting for the role of hypoxia as a trigger for EMT and MET in the following sense. While the full spectrum of mechanisms underlying the induction of EMT remains elusive to date (Wang et al., 2016), it is assumed that tumour-induced hypoxia plays an important role in the process (Imai et al., 2003; Yang et al., 2008; Wang et al., 2016; Petrova et al., 2018). The hypoxic environment in the tumour activates its main effector hypoxia-inducible factor-1 (HIF-1) (Petrova et al., 2018), which in turn activates EMT-TFs like Snail and Twist (Imai et al., 2003; Yang et al., 2008), thus promoting EMT and metastatic phenotypes. A biological model that connects the occurrence of tumour-induced hypoxia with EMT and with angiogenesis via CAFs has recently been proposed in Petrova et al. (2018). The hypothesis is made that rapid tumour growth, which reduces the oxygen concentration in tumour and stroma regions far away from vessels since the diffusion of oxygen is limited to $100-200 \mu \mathrm{m}$, creates hypoxic regions. Epithelial-like cancer cells in these hypoxic regions produce signalling molecules that transform normal fibroblasts as well as other healthy cells in the stroma to CAFs (Zeisberg et al., 2007; Petrova et al., 2018). These CAFs have been shown to produce stiffly aligned ECM. This differently organised ECM is, in turn, hypothesised to induce EMT in premalignant epithelial cells and to support cell migration in breast cancer (Dumont et al., 2013). CAFs have further been shown to promote angiogenesis via the production of vascular endothelial growth factor-C (VEGF), C-X-C motif chemokine 12 (CXCL 12) and basic fibroblast growth factor (FGF-2) (Pietras \& Östman, 2010), making hypoxia an angiogenic stimulus (Carmeliet \& Jain, 2000). Our individual-based spatial modelling framework meets the prerequisites for an extension that includes the above-described biological phenomena. Therefore, we will connect the EMT features currently included in the metastasis framework with the prevalence of tumour-induced acutely and chronically hypoxic regions as well as with angiogenesis in future work.

To create an organ-specific model, we have taken into account differences in the local tumour mi- 
croenvironment of primary and secondary organs in the body in two ways. Firstly, we aligned the relative likelihood of successful secondary spread to the organs in our model to the metastatic transition probabilities of breast cancer from large patient studies (Kuhn Laboratory, 2017). Secondly, we distinguished between the relative differences in ECM density between the organs according to biological measurements in ICRP (2009). We are aware that the variations in ECM density have a minimal influence on the cell movement at the moment, given that their movement is dependent on the ECM gradient rather than the absolute ECM density. However, we are looking to include biomechanical properties such as the ECM stiffness in the model, which has been shown to influence cell behaviour through the activation of intracellular signalling pathways (Kalli \& Stylianopoulos, 2018). At this point, organ-specific and intra-organic differences in ECM will have a larger impact. Also, accounting for organ-specific differences in these two ways is, of course, a simplification of the actual physiology in many ways. For instance, in reality, differences between organs are not limited to the relative densities of their ECM. As explained in detail in Barney et al. (2016), the tissue-specific differences in the tumour microenvironment found in the organs are manifold and only marginally established. They include, for instance, the genetic markers associated with tissue-specific metastasis, the healthy cells typically found in these tissues, the ECM stiffness and its protein composition, and the tissue dimensionality. Also, the tumour microenvironments have been shown to evolve with time, resulting in e.g. pre-metastatic niche formation that has been observed both in mouse models and clinical studies (McAllister \& Weinberg, 2014). Further, these and other features will not only differ between organs but also when considering the same organ in any number of patients. For this reason, it is our goal to include the metastatic programmes of the various organs in our model, once more is known about them. Until then, we will continue using the transition probabilities from large studies as Disibio \& French (2008); Kuhn Laboratory (2017) to differentiate between the relative success of metastatic spread to the various organs.

In Franssen et al. (2019), we investigated the effect of varying the parameters that directly affect the concentration of MT1-MMP and MMP-2 in the system, the haptotactic and diffusive cell movement, the survival probability of cancer cells, and the number of blood vessels at the primary site. In future work, we plan a more in-depth parameter study to identify potential resulting emergent behaviour or significant changes in qualitative results. This way, we aim to extract valuable treatment-related information from the invasion-metastasis model. Additional plans to further develop this modelling framework are elaborated in Franssen et al. (2019). These include extending the model to a third spatial dimension as well as accounting for biomechanical properties, re-seeding, pre-metastatic niche formation, and immune system activation. Moreover, once the modelling framework to account for the mechanistic processed that underlie the invasion-metastasis cascade is sufficiently established and validated, treatment regimes could be modelled. One example is the inclusion of chemotherapy in the framework, especially once we account for angiogenesis, in a similar way to Powathil et al. $(2012,2013)$. Other approaches to modelling chemo-, radio-, nano- and immunotherapy, as well as targeted, hormone and combination therapy, some of which could function as a basis to modelling treatment approaches using this framework, have recently been reviewed by Chamseddine \& Rejniak (2019).

\section{REFERENCES}

Aceto, N., Bardia, A., Miyamoto, D. T., Donaldson, M. C., Wittner, B. S., Spencer, J. A., Yu, M., Pely, A., Engstrom, A., Zhu, H. et al. (2014) Circulating tumor cell clusters are oligoclonal precursors of breast cancer metastasis. Cell, 158(5), 1110-1122.

Andasari, V., Gerisch, A., Lolas, G., South, A. P. \& Chaplain, M. A. J. (2011) Mathematical modeling of cancer cell invasion of tissue: biological insight from mathematical analysis and computational simulation. J. Math. Biol., 63(1), 141-171. 
Anderson, A. R. A. (2005) A hybrid mathematical model of solid tumour invasion: the importance of cell adhesion. Math. Med. Biol., 22(2), 163-186.

Anderson, A. R. A. \& Chaplain, M. A. J. (1998) Continuous and discrete mathematical models of tumor-induced angiogenesis. Bull. Math. Biol., 60(5), 857-899.

Anderson, A. R. A., Chaplain, M. A. J., Newman, E. L., Steele, R. J. C. \& Thompson, A. M. (2000) Mathematical modelling of tumour invasion and metastasis. Comput. Math. Methods Med., 2(2), 129-154.

Armstrong, A. J., Marengo, M. S., Oltean, S., Kemeny, G., Bitting, R. L., Turnbull, J. D., Herold, C. I., Marcom, P. K., George, D. J. \& Garcia-Blanco, M. A. (2011) Circulating tumor cells from patients with advanced prostate and breast cancer display both epithelial and mesenchymal markers. Mol. Cancer Res., 9(8), 9971007.

Banyard, J. \& Bielenberg, D. R. (2015) The role of EMT and MET in cancer dissemination. Connect. Tissue Res., 56(5), 403-413.

Barney, L. E., Jansen, L., Polio, S., Galarza, S., Lynch, M. E. \& Peyton, S. (2016) The predictive link between matrix and metastasis. Curr. Opin. Chem. Eng., 11, 85-93.

Benzekry, S., Tracz, A., Mastri, M., Corbelli, R., Barbolosi, D. \& Ebos, J. M. (2016) Modeling spontaneous metastasis following surgery: an in vivo-in silico approach. Cancer Res., 76(3), 535-547.

Bill, R. \& Christofori, G. (2015) The relevance of EMT in breast cancer metastasis: Correlation or causality? FEBS letters, 589(14), 1577-1587.

Bray, D. (1992) Cell Movements. New York: Garland Publishing.

Burgess, A. E. F., Lorenzi, T., Schofield, P. G., Hubbard, S. F. \& Chaplain, M. A. J. (2017) Examining the role of individual movement in promoting coexistence in a spatially explicit prisoner's dilemma. J. Theor. Biol., 419, 323-332.

Burgess, A. E. F., Schofield, P. G., Hubbard, S. F., Chaplain, M. A. J. \& Lorenzi, T. (2016) Dynamical patterns of coexisting strategies in a hybrid discrete-continuum spatial evolutionary game model. Math. Model. Nat. Phenom., 11(5), 49-64.

Carmeliet, P. \& Jain, R. K. (2000) Angiogenesis in cancer and other diseases. Nature, 407(6801), 249.

Celià-Terrassa, T., Meca-Cortés, Ó., Mateo, F., de Paz, A. M., Rubio, N., Arnal-Estapé, A., Ell, B. J., Bermudo, R., Díaz, A., Guerra-Rebollo, M. \& Lozano, J. (2012) Epithelial-mesenchymal transition can suppress major attributes of human epithelial tumor-initiating cells. J. Clin. Investig., 122(5), 1849-1868.

Chamseddine, I. M. \& Rejniak, K. A. (2019) Hybrid modeling frameworks of tumor development and treatment. Wiley Interdiscip. Rev. Syst. Biol. Med., page e1461.

Chaplain, M. A. J. \& Lolas, G. (2005) Mathematical modelling of cancer cell invasion of tissue: The role of the urokinase plasminogen activation system. Math. Models Methods Appl. Sci., 15(11), 1685-1734.

Cheung, K. J. \& Ewald, A. J. (2016) A collective route to metastasis: Seeding by tumor cell clusters. Science, 352(6282), 167-169.

Cisneros, L. H. \& Newman, T. J. (2014) Quantifying metastatic inefficiency: rare genotypes versus rare dynamics. Phys. Biol., 11(4), 046003.

Collier, I. E., Legant, W., Marmer, B., Lubman, O., Saffarian, S., Wakatsuki, T., Elson, E. \& Goldberg, G. I. (2011) Diffusion of MMPs on the surface of collagen fibrils: the mobile cell surface-collagen substratum interface. PLOS ONE, 6(9), e24029.

Deakin, N. E. \& Chaplain, M. A. J. (2013) Mathematical modeling of cancer invasion: the role of membrane-bound matrix metalloproteinases. Front Oncol, 3, 70.

Disibio, G. \& French, S. W. (2008) Metastatic patterns of cancers: results from a large autopsy study. Archives of pathology \& laboratory medicine, 132(6), 931-939.

Domschke, P., Trucu, D., Gerisch, A. \& Chaplain, M. A. J. (2014) Mathematical modelling of cancer invasion: implications of cell adhesion variability for tumour infiltrative growth patterns. J. Theor. Biol., 361, 41-60.

Domschke, P., Trucu, D., Gerisch, A. \& Chaplain, M. A. J. (2017) Structured models of cell migration incorporating molecular binding processes. J. Math. Biol., 75(6-7), 1517-1561. 
Dongre, A. \& Weinberg, R. A. (2019) New insights into the mechanisms of epithelial-mesenchymal transition and implications for cancer. Nat. Rev. Mol. Cell Biol., 20, 69-84.

Dumont, N., Liu, B., DeFilippis, R. A., Chang, H., Rabban, J. T., Karnezis, A. N., Tjoe, J. A., Marx, J., Parvin, B. \& Tlsty, T. D. (2013) Breast fibroblasts modulate early dissemination, tumorigenesis, and metastasis through alteration of extracellular matrix characteristics. Neoplasia, 15(3), 249-IN7.

Folkman, J. (1990) What is the evidence that tumors are angiogenesis dependent?. J. Natl. Cancer Inst., 82(1), 4-7.

Folkman, J. \& Klagsbrun, M. (1987) Angiogenic factors. Science, 235(4787), 442-447.

Francart, M.-E., Lambert, J., Vanwynsberghe, A. M., Thompson, E. W., Bourcy, M., Polette, M. \& Gilles, C. (2018) Epithelial-mesenchymal plasticity and circulating tumor cells: Travel companions to metastases. Dev. Dyn., 247(3), 432-450.

Franssen, L. C., Lorenzi, T., Burgess, A. E. F. \& Chaplain, M. A. J. (2019) A mathematical framework for modelling the metastatic spread of cancer. Bull. Math. Biol., 81, 1965.

Friedl, P. \& Wolf, K. (2003) Tumour-cell invasion and migration: diversity and escape mechanisms. Nat. Rev. Cancer, 3(5), 362-374.

Gerisch, A. \& Chaplain, M. A. J. (2008) Mathematical modelling of cancer cell invasion of tissue: local and nonlocal models and the effect of adhesion. J. Theor. Biol., 250(4), 684-704.

Guo, W., Keckesova, Z., Donaher, J. L., Shibue, T., Tischler, V., Reinhardt, F., Itzkovitz, S., Noske, A., ZürrerHärdi, U., Bell, G. \& Tam, W. (2012) Slug and Sox9 cooperatively determine the mammary stem cell state. Cell, 148(5), 1015-1028.

Handler, J., Cullis, J., Avanzi, A., Vucic, E. A. \& Bar-Sagi, D. (2018) Pre-neoplastic pancreas cells enter a partially mesenchymal state following transient TGF- $\beta$ exposure. Oncogene, 37(31), 4334-4342.

Hellmann, N., Kolbe, N. \& Sfakianakis, N. (2016) A mathematical insight in the epithelial-mesenchymal-like transition in cancer cells and its effect in the invasion of the extracellular matrix. B. Braz. Math. Soc., 47(1), 397-412.

Huang, R. Y., Wong, M., Tan, T., Kuay, K., Ng, A., Chung, V., Chu, Y., Matsumura, N., Lai, H., Lee, Y. \& Sim, W. (2013) An EMT spectrum defines an anoikis-resistant and spheroidogenic intermediate mesenchymal state that is sensitive to E-cadherin restoration by a Src-kinase inhibitor, saracatinib (AZD0530). Cell Death Dis., 4(11), e915.

Hughes, L., Malone, C., Chumsri, S., Burger, A. M. \& McDonnell, S. (2008) Characterisation of breast cancer cell lines and establishment of a novel isogenic subclone to study migration, invasion and tumourigenicity. Clin. Exp. Metastasis, 25(5), 549-557.

ICRP (2009) Adult Reference Computational Phantoms. Ann. ICRP, 110(39(2)).

Imai, T., Horiuchi, A., Wang, C., Oka, K., Ohira, S., Nikaido, T. \& Konishi, I. (2003) Hypoxia attenuates the expression of E-cadherin via up-regulation of SNAIL in ovarian carcinoma cells. Am. J. Pathol., 163(4), 14371447.

Iwata, K., Kawasaki, K. \& Shigesada, N. (2000) A dynamical model for the growth and size distribution of multiple metastatic tumors. J. Theor. Biol., 203(2), 177-186.

Jie, X.-X., Zhang, X.-Y. \& Xu, C.-J. (2017) Epithelial-to-mesenchymal transition, circulating tumor cells and cancer metastasis: Mechanisms and clinical applications. Oncotarget, 8(46), 81558.

Jolly, M. K., Mani, S. A. \& Levine, H. (2018) Hybrid epithelial/mesenchymal phenotype(s): The 'fittest' for metastasis?. Biochim. Biophys. Acta, Rev. Cancer.

Jolly, M. K., Tripathi, S. C., Somarelli, J. A., Hanash, S. M. \& Levine, H. (2017a) Epithelial/mesenchymal plasticity: how have quantitative mathematical models helped improve our understanding?. Mol. Oncol., 11(7), 739-754.

Jolly, M. K., Ware, K. E., Gilja, S., Somarelli, J. A. \& Levine, H. (2017b) EMT and MET: necessary or permissive for metastasis?. Mol. Oncol., 11(7), 755-769.

Kalli, M. \& Stylianopoulos, T. (2018) Defining the role of solid stress and matrix stiffness in cancer cell proliferation and metastasis. Front. Oncol., 8, 55. 
Krebs, A. M., Mitschke, J., Losada, M. L., Schmalhofer, O., Boerries, M., Busch, H., Boettcher, M., Mougiakakos, D., Reichardt, W., Bronsert, P. \& Brunton, V. (2017) The EMT-activator Zeb1 is a key factor for cell plasticity and promotes metastasis in pancreatic cancer. Nat. Cell Biol., 19(5), 518.

Kröger, C., Afeyan, A., Mraz, J., Eaton, E. N., Reinhardt, F., Khodor, Y. L., Thiru, P., Bierie, B., Ye, X., Burge, C. B. \& Weinberg, R. (2019) Acquisition of a hybrid E/M state is essential for tumorigenicity of basal breast cancer cells. Proc. Natl. Acad. Sci., page 201812876.

Kuhn Laboratory (2017) Breast Cancer Progression Models. http://kuhn.usc.edu/breast_cancer/. Accessed: 22-05-2019.

Lambert, A. W., Pattabiraman, D. R. \& Weinberg, R. A. (2017) Emerging biological principles of metastasis. Cell, 168(4), 670-691.

Lovisa, S., LeBleu, V. S., Tampe, B., Sugimoto, H., Vadnagara, K., Carstens, J. L., Wu, C.-C., Hagos, Y., Burckhardt, B. C., Pentcheva-Hoang, T. \& Nischal, H. (2015) Epithelial-to-mesenchymal transition induces cell cycle arrest and parenchymal damage in renal fibrosis. Nat. Med., 21(9), 998.

Luzzi, K. J., MacDonald, I. C., Schmidt, E. E., Kerkvliet, N., Morris, V. L., Chambers, A. F. \& Groom, A. C. (1998) Multistep nature of metastatic inefficiency: dormancy of solitary cells after successful extravasation and limited survival of early micrometastases. Am. J. Pathol., 153(3), 865-873.

Margarit, D. H. \& Romanelli, L. (2016) A mathematical model of absorbing Markov chains to understand the routes of metastasis. Biomath., 5(1), 1607281.

McAllister, S. S. \& Weinberg, R. A. (2014) The tumour-induced systemic environment as a critical regulator of cancer progression and metastasis. Nat. Cell Biol., 16(8), 717-727.

McDougall, S. R., Watson, M. G., Devlin, A. H., Mitchell, C. A. \& Chaplain, M. A. J. (2012) A hybrid discretecontinuum mathematical model of pattern prediction in the developing retinal vasculature. Bull. Math. Biol., 74(10), 2272-2314.

Meng, S., Tripathy, D., Frenkel, E. P., Shete, S., Naftalis, E. Z., Huth, J. F., Beitsch, P. D., Leitch, M., Hoover, S., Euhus, D., Haley, B., Morrison, L., Fleming, T. P., Herlyn, D., Terstappen, L. W., Fehm, T., Tucker, T. F., Lane, N., Wang, J. \& Uhr, J. W. (2004) Circulating tumor cells in patients with breast cancer dormancy. Clin. Cancer Res., 10(24), 8152-8162.

Micalizzi, D. S., Farabaugh, S. M. \& Ford, H. L. (2010) Epithelial-mesenchymal transition in cancer: Parallels between normal development and tumor progression. J. Mammary Gland Biol. Neoplasia, 15(2), 117-134.

Milo, R., Jorgensen, P., Moran, U., Weber, G. \& Springer, M. (2009) BioNumbers-the database of key numbers in molecular and cell biology. Nucleic Acids Res., 38(suppl_1), D750-D753. Accessed: 22-10-2017.

Morton, K. W. \& Mayers, D. F. (2005) Numerical solution of partial differential equations: an introduction, pages 151-193. Cambridge university press.

NCI (2015) Cell lines in the in vitro screen. Accessed: 15-07-2019.

Nurmenniemi, S., Sinikumpu, T., Alahuhta, I., Salo, S., Sutinen, M., Santala, M., Risteli, J., Nyberg, P. \& Salo, T. (2009) A novel organotypic model mimics the tumor microenvironment. Am. J. Pathol., 175(3), 1281-1291.

Obenauf, A. C. \& Massagué, J. (2015) Surviving at a distance: organ-specific metastasis. Trends Cancer, 1(1), $76-91$.

Ocaña, O. H., Córcoles, R., Fabra, Á., Moreno-Bueno, G., Acloque, H., Vega, S., Barrallo-Gimeno, A., Cano, A. \& Nieto, M. A. (2012) Metastatic colonization requires the repression of the epithelial-mesenchymal transition inducer Prrx1. Cancer cell, 22(6), 709-724.

Pastushenko, I. \& Blanpain, C. (2018) EMT transition states during tumor progression and metastasis. Trends Cell Biol., 29(3), 212-226.

Petrova, V., Annicchiarico-Petruzzelli, M., Melino, G. \& Amelio, I. (2018) The hypoxic tumour microenvironment. Oncogenesis, 7(1), 10.

Pietras, K. \& Östman, A. (2010) Hallmarks of cancer: interactions with the tumor stroma. Exp. Cell Res., 316(8), 1324-1331.

Powathil, G. G., Adamson, D. J. A. \& Chaplain, M. A. J. (2013) Towards predicting the response of a solid tumour 
to chemotherapy and radiotherapy treatments: clinical insights from a computational model. PLoS Comput. Biol., 9(7), e1003120.

Powathil, G. G., Gordon, K. E., Hill, L. A. \& Chaplain, M. A. J. (2012) Modelling the effects of cell-cycle heterogeneity on the response of a solid tumour to chemotherapy: biological insights from a hybrid multiscale cellular automaton model. J. Theor. Biol., 308, 1-19.

Puram, S. V., Tirosh, I., Parikh, A. S., Patel, A. P., Yizhak, K., Gillespie, S., Rodman, C., Luo, C. L., Mroz, E. A., Emerick, K. S. \& Deschler, D. (2017) Single-cell transcriptomic analysis of primary and metastatic tumor ecosystems in head and neck cancer. Cell, 171(7), 1611-1624.

Roche, J. (2018) The epithelial-to-mesenchymal transition in cancer. .

Ruscetti, M., Quach, B., Dadashian, E. L., Mulholland, D. J. \& Wu, H. (2015) Tracking and functional characterization of epithelial-mesenchymal transition and mesenchymal tumor cells during prostate cancer metastasis. Cancer Res., 75(13), 2749-2759.

Scott, J. G., Basanta, D., Anderson, A. R. A. \& Gerlee, P. (2013) A mathematical model of tumour self-seeding reveals secondary metastatic deposits as drivers of primary tumour growth. J. R. Soc. Interface, 10(82), 20130011.

Sfakianakis, N., Kolbe, N., Hellmann, N. \& Lukáčová-Medvid’ová, M. (2017) A multiscale approach to the migration of cancer stem cells: Mathematical modelling and simulations. Bull. Math. Biol., 79(1), 209-235.

Sfakianakis, N., Madzvamuse, A. \& Chaplain, M. A. J. (2018) A hybrid multiscale model for cancer invasion of the extracellular matrix. arXiv:1805.10541 [Preprint]. Accessed: 25-08-2019.

Stéphanou, A., McDougall, S. R., Anderson, A. R. A. \& Chaplain, M. A. J. (2006) Mathematical modelling of the influence of blood rheological properties upon adaptative tumour-induced angiogenesis. Math. Comput. Model., 44(1-2), 96-123.

Stokes, C., Rupnick, M., Williams, S. \& Lauffenburger, D. (1990) Chemotaxis of human microvessel endothelial cells in response to acidic fibroblast growth factor.. Lab. Invest., 63(5), 657-668.

Thiery, J. P. \& Lim, C. T. (2013) Tumor dissemination: an EMT affair. Cancer cell, 23(3), 272-273.

Tsai, J. H., Donaher, J. L., Murphy, D. A., Chau, S. \& Yang, J. (2012) Spatiotemporal regulation of epithelialmesenchymal transition is essential for squamous cell carcinoma metastasis. Cancer cell, 22(6), 725-736.

Vajtai, R. (2013) Springer handbook of nanomaterials. Springer Science \& Business Media.

Valastyan, S. \& Weinberg, R. A. (2011) Tumor metastasis: molecular insights and evolving paradigms. Cell, 147(2), 275-292.

Vega, S., Morales, A. V., Ocaña, O. H., Valdés, F., Fabregat, I. \& Nieto, M. A. (2004) Snail blocks the cell cycle and confers resistance to cell death. Genes Dev., 18(10), 1131-1143.

Wang, X., Yu, M., Zhao, K., He, M., Ge, W., Sun, Y., Wang, Y., Sun, H. \& Hu, Y. (2016) Upregulation of MiR-205 under hypoxia promotes epithelial-mesenchymal transition by targeting ASPP2. Cell Death Dis., 7(12), e2517.

Yang, M.-H., Wu, M.-Z., Chiou, S.-H., Chen, P.-M., Chang, S.-Y., Liu, C.-J., Teng, S.-C. \& Wu, K.-J. (2008) Direct regulation of TWIST by HIF-1 $\alpha$ promotes metastasis. Nat. Cell Biol., 10(3), 295.

Ye, X., Tam, W. L., Shibue, T., Kaygusuz, Y., Reinhardt, F., Eaton, E. N. \& Weinberg, R. A. (2015) Distinct EMT programs control normal mammary stem cells and tumour-initiating cells. Nature, 525(7568), 256.

Yu, M., Bardia, A., Wittner, B. S., Stott, S. L., Smas, M. E., Ting, D. T., Isakoff, S. J., Ciciliano, J. C., Wells, M. N., Shah, A. M., \& Concannon, K. F. (2013) Circulating breast tumor cells exhibit dynamic changes in epithelial and mesenchymal composition. Science, 339(6119), 580-584.

Zeisberg, E. M., Potenta, S., Xie, L., Zeisberg, M. \& Kalluri, R. (2007) Discovery of endothelial to mesenchymal transition as a source for carcinoma-associated fibroblasts. Cancer Res., 67(21), 10123-10128. 


\section{Appendices}

\section{A. Previously established metastasis modelling framework}

The general spatial modelling framework of the metastatic spread of cancer developed in Franssen et al. (2019) is appended here for ease of accessibility. This previous modelling framework underlies the changes to the model introduced in the main body of this paper in Section 3. These changes have the purpose of including EMT, MET as well as the third partial-EMT cancer cell phenotype into the existing framework. Further changes are the differentiation of organ tissue via an organ-specific initial ECM density as well as the inclusion of cell death and dormancy at secondary sites in the body.

To account for cancer cell metastasis in a spatially explicit manner, we consider $G+1$ spatial domains. These consist of the spatial domain representing the primary tumour site, $\Omega_{\mathrm{p}} \subset \mathbb{R}^{2}$, as well as the $G \in \mathbb{N}$ spatial domains representing the sites of potential secondary metastatic spread, $\Omega_{\mathrm{s}}^{a} \subset \mathbb{R}^{2}$, where $a=1,2, \ldots, G$. In these spatial domains, we represent the MMP-2 concentration and the ECM density at positions $\vec{x}$ at time $t$ by the continuous functions $m(t, \vec{x})$ and $w(t, \vec{x})$, respectively, while capturing the spatiotemporal evolution of epithelial-like and mesenchymal-like cancer cells individually. We model the local cancer cell invasion by expanding the modelling approach used in Anderson \& Chaplain (1998); Anderson et al. (2000) to our specific biological problem. However, we include a second cancer cell phenotype and also additionally consider MT1-MMP, which is taken to be bound to the membranes of the mesenchymal-like cancer cells and thus follows their discrete spatiotemporal dynamics. We designate locations in the primary spatial domain to function as entry points into the vasculature and, similarly, impose a spatial map of exit locations from the vasculature onto the secondary metastatic domains. This allows cancer cells to travel from the primary tumour site to secondary sites via blood vessels.

We next consider one key step of the invasion-metastasis cascade after the other. To make the key steps more recognisable, we begin each paragraph by printing the description of the corresponding step in the invasion-metastasis cascade ( $c f$. Section 2 in Franssen et al. (2019)) in bold. Further, the same step descriptions can be found on the left of the flowchart in Figure 4 in Franssen et al. (2019). This highlights which parts of our model correspond to which sections in the text.

Local cancer cell invasion We adopt a discrete cell approach where the movement of cancer cells from grid point to grid point is accounted for by movement probabilities consisting of an unbiased component ( $c f$. random motion) and a biased component proportional to gradients in the ECM ( $c f$. haptotaxis). This way, we obtain the movement probabilities of the individual epithelial-like and mesenchymal-like cancer cells in equation (3.3) with $k=\mathrm{E}, \mathrm{M}$. Modelling the cancer cells individually allows us to track the evolution of single epithelial-like and mesenchymal-like cancer cells with different phenotypes, as well as their evolution.

The model we have described so far accounts for the movement of the cancer cells only. We thus need to additionally account for the proliferation of cancer cells in our model. The two cancer cell types included in our model proliferate at different frequencies. The more proliferative epithelial-like cancer cells perform mitosis after time interval $T_{\mathrm{E}}$, the less proliferative mesenchymal-like cell types after $T_{\mathrm{M}}$ (with $T_{\mathrm{M}}>T_{\mathrm{E}}$ ). When proliferating, the cancer cells pass on their respective phenotype as well as their location so that a proliferating cancer cell is replaced by two daughter cells after the proliferative step has been performed. However, to account for competition for space and resources, the cancer cells on the respective grid point do not proliferate if there are $\mathscr{Q} \in \mathbb{N}$ cancer cells on a grid point at the time of proliferation.

With reference to the flowchart shown in Figure 4 in Franssen et al. (2019), the part of our approach 
described so far corresponds to Movement, EMT \& cell proliferation, depicted in the upper region of the flowchart.

The mesenchymal-like cancer cells in our model have the ability to express diffusible MMP-2. The MMP-2 concentration $m(t, \vec{x})$ hence develops according to the equation:

$$
\frac{\partial m}{\partial t}=\overbrace{D_{m} \nabla^{2} m}^{\text {diffusion }}+\overbrace{\Theta c_{\mathrm{M}_{n}}}^{\text {expression }}-\overbrace{\Lambda m}^{\text {decay }},
$$

along with zero-flux boundary conditions. Here, $c_{\mathrm{M}_{n}}, n=0,1,2, \ldots, \mathscr{Q}$, indicates the presence of up to $\mathscr{Q}$ mesenchymal-like cancer cells at a given position ( $c f$. Stéphanou et al. (2006); McDougall et al. (2012)). $D_{m}>0$ is the constant MMP-2 diffusion coefficient, $\Theta>0$ is the constant rate of MMP- 2 concentration provided by mesenchymal-like cancer cells, and $\Lambda>0$ is the constant rate at which MMP-2 decays. Consequently, $\Theta c_{\mathrm{M}_{n}}$ represents the local expression of MMP-2 by the $c_{\mathrm{M}_{n}}$ mesenchymal-like cancer cells. Note that the mesenchymal-like cancer cells also express MT1-MMP. However, MT1-MMP acts locally only where it is bound to the cancer cell membrane and its spatiotemporal evolution is hence congruent to that of the mesenchymal-like cancer cells. Therefore, we do not include a separate equation.

The diffusible MMP-2 degrades the ECM with a degradation rate of $\Gamma_{2}>0$. The MT1-MMP expressed on the membrane of the mesenchymal-like cancer cells also degrades the ECM, which is expressed through the degradation rate $\Gamma_{1}>0$. Hence, given that we are disregarding ECM-remodelling for simplicity, the evolution of the ECM density $w(t, \vec{x})$ is governed by the following PDE:

$$
\frac{\partial w}{\partial t}=-\overbrace{\left(\Gamma_{1} c_{\mathrm{M}_{n}}+\Gamma_{2} m\right) w}^{\text {degradation }},
$$

along with zero-flux boundary conditions. As before, $c_{\mathrm{M}_{n}}, n=0,1,2, \ldots, \mathscr{Q}$, indicates the presence of up to $\mathscr{Q}$ mesenchymal-like cancer cells at a given position ( $c f$. Stéphanou et al. (2006); McDougall et al. (2012)).

Since the continuous evolution of the MMP-2 concentration and of the ECM density is governed by equations (A.1) and (A.2), while the spatiotemporal evolution of the cancer cells (and, intrinsically, of the membrane-bound MT1-MMP) is captured by an individual-based model, $c f$. equation (3.3), we model cancer cell invasion in a hybrid-discrete continuum approach of the kind pioneered by Anderson \& Chaplain (1998) in their tumour-angiogenesis model. This approach was subsequently also used to model tissue invasion by cancer cells (Anderson et al., 2000; Anderson, 2005) and spatial evolutionary games (Burgess et al., 2016, 2017).

Intravasation With the model setup we have described so far, the cancer cells can invade the tissue locally in the primary spatial domain but cannot reach the spatially separated secondary domains. To allow for metastatic spread, we account for the connection of the primary spatial domain to the secondary spatial domains by incorporating blood vessels in our modelling framework. Examples of primary and secondary domains are presented in Figure 6. To represent the entry points into the blood vessels, a number of $U_{\mathrm{P}} \in \mathbb{N}_{0}$ normal blood vessels as well as $V_{\mathrm{P}} \in \mathbb{N}_{0}$ ruptured blood vessels are distributed on the primary grid. The normal blood vessels take the size of one grid point, while ruptured vessels consist of a group of $A^{b} \in \mathbb{N}$, where $b=1,2, \ldots, V_{\mathrm{P}}$, adjacent grid points and can thus have different shapes. Each secondary grid $\Omega_{\mathrm{s}}^{a}$ also has, respectively, $U_{\mathrm{s}}^{a} \in \mathbb{N}$ normal blood vessels, where $a=1,2, \ldots, G$ as before, 
that take the form of a single grid point each. On the primary grid, the grid points where the vessels are located allow the cancer cells to intravasate, while the respective grid points on the secondary grid allow for extravasation.

If, by the movement submodel described in Appendix B of Franssen et al. (2019), a cancer cell on the primary grid is placed on a grid point that represents a blood vessel, it may leave the grid and enter the vasculature. Whether or not a cancer cell can successfully intravasate depends both on its own phenotype and on the type of vessel it is placed on.

Whenever a mesenchymal-like cancer cell is moved to a grid point $\left(x_{i}, y_{j}\right) \in \Omega_{\mathrm{p}}{ }^{1}$, on which a normal single blood vessel is located, it will successfully enter the vasculature. Further, to represent collective invasion in the form of co-presence of mesenchymal-like and epithelial-like cancer cells, cancer cells of any type on the four neighbouring primary grid points $\left(x_{i+1}, y_{j}\right),\left(x_{i-1}, y_{j}\right),\left(x_{i}, y_{j+1}\right)$ and $\left(x_{i}, y_{j-1}\right)$ are forced into the vasculature together with the mesenchymal-like cancer cell on $\left(x_{i}, y_{j}\right)$. Hence, a mesenchymal-like cancer cell moving to a grid point on which a normal blood vessel is located results in either a single mesenchymal-like cancer cell or a cluster consisting of up to $5 \mathscr{Q}$ cancer cells of any phenotype intravasating. However, if an epithelial-like cancer cell is moved to a grid point $\left(x_{i}, y_{j}\right) \in \Omega_{\mathrm{P}}$ where a normal single vessel is located without a mesenchymal-like cell being present there, the epithelial-like cancer cell will not intravasate and the grid point $\left(x_{i}, y_{j}\right)$ will be treated like any other grid point. This is to model the fact that epithelial-like cancer cells have been shown to be unable to actively intravasate on their own.

Further, a cancer cell on the primary grid can move to one of the grid points where a ruptured vessel is located. Contrary to the above-described scenario of entering a normal vessel, a cancer cell of any type, which is placed on a grid point representing part of a ruptured vessel, can enter the circulation. The respective cancer cell takes with it any other cancer cells residing both on the grid points representing the ruptured blood vessel and on the regular grid points bordering the ruptured vessel. Biologically, the fact that cancer cells of any phenotype can intravasate mirrors that these blood vessels are already ruptured due to trauma or pressure applied by the expanding tumour, making the requirement of MDE-mediated degradation of the vessel wall redundant. The fact that other cancer cells on bordering grid points will enter the circulation together with cancer cells placed on grid points representing blood vessels captures some degree of the cell-cell adhesion found in collectively invading cancer cell clusters.

Travel through the vasculature If a cancer cell of either phenotype or a cluster of cancer cells successfully enters the vasculature either through a ruptured or a normal vessel, it will be removed from the primary grid and moved to the vasculature. Cancer cells and cancer cell clusters remain in the vasculature for some time $T_{V} \in \mathbb{N}$, which biologically represents the average time the cancer cells spend in the blood system. Any cancer cells that enter a particular vessel at the same time are treated as one cluster and hence as a single entity once they are located in the vasculature. However, each cancer cell that is part of a cancer cell cluster disaggregates from its cluster with some probability $\mathscr{P}_{d}$ after $\left\lceil\frac{T_{V}}{2}\right\rceil$ time steps. At the end of the time period $T_{V}$, the single cancer cells and the remaining cancer cell clusters are removed from the simulation unless they are randomly determined to survive. The survival probability is $\mathscr{P}_{S}$ for single cancer cells and $\mathscr{P}_{C}$ for cancer cell clusters.

Extravasation Any surviving cancer cells and cancer cell clusters are placed on one of the $G$ secondary grids $\Omega_{\mathrm{S}}^{a}$ with probability $\mathscr{E}_{1}, \mathscr{E}_{2}, \ldots, \mathscr{E}_{G}$, where $\sum_{a=1}^{G} \mathscr{E}_{a}=1$. Also, on each specific secondary grid, the cancer cells extravasate through one of the randomly chosen $U_{\mathrm{s}}^{a}$ grid points that represent a blood vessel with equal probability. If the respective grid point cannot accommodate all of the en-

\footnotetext{
${ }^{1}$ The notation $\left(x_{i}, y_{j}\right) \in \Omega_{\mathrm{P}}$ is a result of the discretisation of the grids in our model, as described in detail in Appendix B of Franssen et al. (2019).
} 
38 of 40

tering cancer cells without violating the preferred carrying capacity $\mathscr{Q}$, the remaining cancer cells are randomly distributed onto the four non-diagonally neighbouring grid points until these are filled to the preferred carrying capacity $\mathscr{Q}$. If there are further cancer cells to be placed onto the respective grid point at this instance, such cancer cells are killed to capture the influence of competition for space in combination with vascular flow dynamics.

Metastatic growth If and when cancer cells reach a secondary grid, they behave (i.e. replicate, move, produce MDEs etc.) there according to the same rules as on the primary grid, as indicated on the bottom of the flowchart in Figure 4 of Franssen et al. (2019). 


\section{B. Parameter settings used in the simulations}

Table A.1: Parameter settings used in the simulations. In the first column, non-dimensional parameters are indicated by upper-case notation. Corresponding dimensional parameters are stated in brackets using lower-case notation. In the fourth column, we reference other mathematical modelling papers in brackets and biological papers without brackets. Epithelial-like, partial-EMT and mesenchymal-like cancer cells are represented by the acronyms ECC, PCC and MCC, respectively.

\begin{tabular}{|c|c|c|c|c|}
\hline & Description & $\begin{array}{l}\text { Non-dimen- } \\
\text { sional value }\end{array}$ & $\begin{array}{l}\text { Biological reference } \\
\text { (Modelling reference) }\end{array}$ & $\begin{array}{l}\text { Original value } \\
\text { from cited source }\end{array}$ \\
\hline$\Delta t$ & Time step & $1 \times 10^{-3}$ & & $40 \mathrm{~s}$ \\
\hline $\begin{array}{l}\Delta x \\
\Delta y\end{array}$ & Space step & $5 \times 10^{-3}$ & $\begin{array}{l}\text { Breast cell diameter in } \\
\text { Vajtai (2013) }\end{array}$ & $1 \times 10^{-3} \mathrm{~cm}$ \\
\hline $\begin{array}{l}D_{\mathrm{E}} \\
\left(d_{\mathrm{E}}\right)\end{array}$ & ECC diffusion coefficient & $1 \times 10^{-4}$ & $\begin{array}{l}\text { Bray (1992) } \\
\text { (Anderson \& Chaplain (1998)) } \\
\text { (Deakin \& Chaplain (2013)) }\end{array}$ & $1 \times 10^{-10} \mathrm{~cm}^{2} \mathrm{~s}^{-1}$ \\
\hline $\begin{array}{l}D_{\mathrm{E} / \mathrm{M}} \\
\left(d_{\mathrm{E} / \mathrm{M}}\right)\end{array}$ & PCC diffusion coefficient & $2.5 \times 10^{-4}$ & $\begin{array}{l}\text { Bray (1992) } \\
\text { (Anderson \& Chaplain (1998)) } \\
\text { (Deakin \& Chaplain (2013)) }\end{array}$ & $1 \times 10^{-10} \mathrm{~cm}^{2} \mathrm{~s}^{-1}$ \\
\hline $\begin{array}{l}D_{\mathrm{M}} \\
\left(d_{\mathrm{M}}\right)\end{array}$ & MCC diffusion coefficient & $5 \times 10^{-4}$ & $\begin{array}{l}\text { Bray (1992) } \\
\text { (Anderson \& Chaplain (1998)) } \\
\text { (Deakin \& Chaplain (2013)) }\end{array}$ & $1 \times 10^{-10} \mathrm{~cm}^{2} \mathrm{~s}^{-1}$ \\
\hline $\begin{array}{l}\Phi_{\mathrm{E}} \\
\left(\phi_{\mathrm{E}}\right)\end{array}$ & $\begin{array}{l}\text { ECC haptotactic } \\
\text { sensitivity coefficient }\end{array}$ & $5 \times 10^{-5}$ & $\begin{array}{l}\text { Stokes et al. (1990) } \\
\text { (Anderson \& Chaplain (1998)) }\end{array}$ & $2.6 \times 10^{3} \mathrm{~cm}^{2} \mathrm{M}^{-1} \mathrm{~s}^{-1}$ \\
\hline $\begin{array}{l}\Phi_{\mathrm{E} / \mathrm{M}} \\
\left(\phi_{\mathrm{E} / \mathrm{M}}\right)\end{array}$ & $\begin{array}{l}\text { PCC haptotactic } \\
\text { sensitivity coefficient }\end{array}$ & $1 \times 10^{-3}$ & $\begin{array}{l}\text { Stokes et al. (1990) } \\
\text { (Anderson \& Chaplain (1998)) }\end{array}$ & $2.6 \times 10^{3} \mathrm{~cm}^{2} \mathrm{M}^{-1} \mathrm{~s}^{-1}$ \\
\hline $\begin{array}{l}\Phi_{\mathrm{M}} \\
\left(\phi_{\mathrm{M}}\right) \\
\end{array}$ & $\begin{array}{l}\text { MCC haptotactic } \\
\text { sensitivity coefficient }\end{array}$ & $2 \times 10^{-3}$ & $\begin{array}{l}\text { Stokes et al. (1990) } \\
\text { (Anderson \& Chaplain (1998)) }\end{array}$ & $2.6 \times 10^{3} \mathrm{~cm}^{2} \mathrm{M}^{-1} \mathrm{~s}^{-1}$ \\
\hline $\begin{array}{l}D_{\mathrm{m}} \\
\left(d_{\mathrm{m}}\right)\end{array}$ & $\begin{array}{l}\text { MMP-2 diffusion } \\
\text { coefficient }\end{array}$ & $1 \times 10^{-3}$ & Collier et al. (2011) & $1 \times 10^{-9} \mathrm{~cm}^{2} \mathrm{~s}^{-1}$ \\
\hline $\begin{array}{l}\Theta_{E} \\
\left(\theta_{E}\right) \\
\end{array}$ & $\begin{array}{l}\text { MMP- } 2 \text { production rate } \\
\text { by ECCs }\end{array}$ & 0 & Estimated & \\
\hline $\begin{array}{l}\Theta_{E / M} \\
\left(\theta_{E / M}\right)\end{array}$ & $\begin{array}{l}\text { MMP- } 2 \text { production rate } \\
\text { by PCCs }\end{array}$ & 0.1 & Estimated & \\
\hline $\begin{array}{l}\Theta_{M} \\
\left(\theta_{M}\right) \\
\end{array}$ & $\begin{array}{l}\text { MMP- } 2 \text { production rate } \\
\text { by MCCs }\end{array}$ & 0.195 & Estimated & \\
\hline $\begin{array}{l}\Lambda \\
(\lambda)\end{array}$ & MMP-2 decay rate & 0.1 & $\begin{array}{l}\text { Estimated in } \\
\text { (Deakin \& Chaplain, 2013) }\end{array}$ & $2.5 \times 10^{-6} \mathrm{~s}^{-1}$ \\
\hline $\begin{array}{l}\Gamma_{1} \\
\left(\gamma_{1}\right)\end{array}$ & $\begin{array}{l}\text { ECM degradation } \\
\text { rate by MT1-MMP }\end{array}$ & 1 & $\begin{array}{l}\text { Based on } \\
\text { (Deakin \& Chaplain, 2013) }\end{array}$ & $1 \times 10^{-4} \mathrm{~s}^{-1}$ \\
\hline $\begin{array}{l}\Gamma_{2} \\
\left(\gamma_{2}\right)\end{array}$ & $\begin{array}{l}\text { ECM degradation } \\
\text { rate by MMP-2 }\end{array}$ & 1 & $\begin{array}{l}\text { Based on } \\
\text { (Anderson et al., 2000) }\end{array}$ & $1 \times 10^{-4} \mathrm{M}^{-1} \mathrm{~s}^{-1}$ \\
\hline
\end{tabular}




\begin{tabular}{|c|c|c|c|c|}
\hline$T_{V}$ & $\begin{array}{l}\text { Time CTCs spend in the } \\
\text { vasculature }\end{array}$ & 0.18 & Meng et al. (2004) & $7.2 \times 10^{3} \mathrm{~s}$ \\
\hline$\underline{T_{\mathrm{E}}}$ & ECC doubling time & 2 & Milo et al. (2009); NCI (2015) & $8 \times 10^{4} \mathrm{~s}$ \\
\hline$T_{\mathrm{E} / \mathrm{M}}$ & PCC doubling time & 3 & Milo et al. (2009); NCI (2015) & $1.2 \times 10^{5} \mathrm{~s}$ \\
\hline$T_{\mathrm{M}}$ & MCC doubling time & 6 & $\begin{array}{l}\text { Milo et al. (2009); NCI (2015) } \\
\text { Hughes et al. (2008) }\end{array}$ & $2.4 \times 10^{5} \mathrm{~s}$ \\
\hline $\mathscr{P}_{\delta}^{\Omega_{S}^{1,2,3}}$ & $\begin{array}{l}\text { Probability no cell proliferation } \\
\text { when due on } \Omega_{\mathrm{S}}^{1,2,3}\end{array}$ & 0.5 & Estimated & \\
\hline $\mathscr{P}_{D}^{\Omega_{\mathrm{S}}^{1,2,3}}$ & $\begin{array}{l}\text { Probability cell death before } \\
\text { proliferation on } \Omega_{\mathrm{S}}^{1,2,3}\end{array}$ & 0.05 & Estimated & \\
\hline $\mathscr{P}_{\mathrm{E}}=\mathscr{P}_{\mathrm{M}}$ & $\begin{array}{l}\text { Epithelial/mesenchymal } \\
\text { CTC survival probability }\end{array}$ & $2 \times 10^{-4}$ & Luzzi et al. (1998) & \\
\hline $\mathscr{P}_{\mathrm{E} / \mathrm{M}}$ & $\begin{array}{l}\text { Partial-EMT CTC } \\
\text { survival probability }\end{array}$ & $6 \times 10^{-4}$ & Luzzi et al. (1998) & \\
\hline $\mathscr{P}_{C}$ & $\begin{array}{l}\text { CTC cluster survival } \\
\text { probability }\end{array}$ & $2.5 \times 10^{-2}$ & $\begin{array}{l}\text { Luzzi et al. (1998) } \\
\text { Aceto et al. (2014) }\end{array}$ & \\
\hline $\mathscr{P}_{\mathrm{S}}^{1}$ & $\begin{array}{l}\text { Probability for surviving cells } \\
\text { to extravasate to bones }\end{array}$ & $\sim 0.5461$ & Kuhn Laboratory (2017) & $\sim 0.5461$ \\
\hline $\mathscr{P}_{\mathrm{S}}^{2}$ & $\begin{array}{l}\text { Probability for surviving cells } \\
\text { to extravasate to lungs }\end{array}$ & $\sim 0.2553$ & Kuhn Laboratory (2017) & $\sim 0.2553$ \\
\hline $\mathscr{P}_{\mathrm{S}}^{3}$ & $\begin{array}{l}\text { Probability for surviving cells } \\
\text { to extravasate to liver }\end{array}$ & $\sim 0.1986$ & Kuhn Laboratory (2017) & $\sim 0.1986$ \\
\hline $\begin{array}{l}\mathscr{P} \mathrm{M} \\
\mathrm{EMT}\end{array}$ & Probability for full EMT on $\Omega_{P}$ & $1 \times 10^{-2}$ & Estimated & \\
\hline $\begin{array}{l}\mathscr{P} \text { E/M } \\
\text { EMT }\end{array}$ & $\begin{array}{l}\text { Probability for partial EMT } \\
\text { on } \Omega_{P}\end{array}$ & $2 \times 10^{-2}$ & Estimated & \\
\hline $\begin{array}{l}\mathscr{P P} \mathrm{E} / \mathrm{M}^{*} \\
\text { EMT }\end{array}$ & $\begin{array}{l}\text { Additional probability for partial } \\
\text { EMT on } \Omega_{P} \text { at tumour edge }\end{array}$ & 0.15 & Estimated & \\
\hline $\mathscr{P}_{\mathrm{MET}}^{\mathrm{E}}$ & $\begin{array}{l}\text { Probability for partial MET } \\
\text { on } \Omega_{\mathrm{S}}^{1,2,3}\end{array}$ & 0.5 & Estimated & \\
\hline$w(0, \vec{x}), \vec{x} \in \Omega_{\mathrm{P}}$ & Breast initial ECM density & 1 & ICRP (2009) & $1.020 \mathrm{~g} \mathrm{~cm}^{-3}$ \\
\hline$w(0, \vec{x}), \vec{x} \in \Omega_{\mathrm{S}}^{1}$ & Bone initial ECM density & 0.9608 & ICRP (2009) & $0.980 \mathrm{~g} \mathrm{~cm}^{-3}$ \\
\hline$w(0, \vec{x}), \vec{x} \in \Omega_{\mathrm{S}}^{2}$ & Lung initial ECM density & 1.0392 & ICRP (2009) & $1.060 \mathrm{~g} \mathrm{~cm}^{-3}$ \\
\hline$w(0, \vec{x}), \vec{x} \in \Omega_{\mathrm{S}}^{3}$ & Liver initial ECM density & 1.0294 & ICRP (2009) & $1.050 \mathrm{~g} \mathrm{~cm}^{-3}$ \\
\hline
\end{tabular}

\section{Funding}

This work was supported by the Engineering and Physical Sciences Research Council (EPSRC) [to L.C.F.]; EPSRC Grant No. EP/N014642/1 (EPSRC Centre for Multiscale Soft Tissue Mechanics With Application to Heart \& Cancer) [to M.A.J.C.]. 